\title{
Assessing knowledge of suicide: a systematic review of available instruments
}

A. Porte ${ }^{1,2,3,4 *}$, M. Wathelet $t^{3,4,5}$, P. Grandgenèvre ${ }^{1,2,3,4}$, G. Vaiva $a^{1,2,3,4,6}$, C-E. Notredame $e^{1,2,3,4}$

${ }^{1}$ PSY Lab, Lille Neuroscience \& Cognition Centre, INSERM U1172, Lille University, F59000 Lille, France

${ }^{2}$ CHU Lille, Psychiatry Department, F-59000 Lille, France

${ }^{3}$ Groupement d'Étude et de Prévention du Suicide, F-86280 Saint-Benoît, France

${ }^{4}$ Papageno Program, F-59000 Lille, France

${ }^{5}$ CHU Lille, Public Health department, F-59000 Lille, France

${ }^{6}$ Centre National de Ressources et de Résilience, F-59000 Lille, France

* Corresponding author:

Dr Amélie Porte

Hôpital Fontan, CHU Lille

2, rue André Verhaeghe, F-59000 Lille, France

Tel: +33 320445962

e-mail: amelie.porte@chru-lille.fr 


\begin{abstract}
Objective. Literacy of suicide is the corner stone of numerous prevention programs but is a difficult construct to appraise. To bring methodological and epistemological clarifications, we aimed to investigate the actual content and psychometric properties of the available tools designed to inquire knowledge of suicide.
\end{abstract}

Methods. We conducted a systematic review of the literature. Electronic databases were searched for questionnaire assessing literacy, attitudes, knowledge or misconceptions about suicide. After checking the quality of validation procedures, we exhaustively collected the psychometric properties of the scales. Contents were submitted to a qualitative thematic analysis.

Results. We identified 18 unique instruments from 48 papers. On the metrological level, general poor to fair compliance with validation standards and variability of psychometric properties stand out as the most prominent results. As regards to the constructs that instruments appraise, we derived 6 thematic categories of knowledge: epidemiology, consequences of media coverage and 4 common myths about the presumed monocausality, unpredictability, harmlessness and unpreventability of suicidal behaviors. Overall, five scales emerge as robust and/or valid enough tools to probe knowledge of suicide.

Limitations. We chose selection criteria based on a priori conceptions of literacy. This may have restricted the scope of retrievable scales and limited our inferences about what is explored under the labels "knowledge" or "literacy".

Conclusions. To design refined instruments about literacy of suicide, scholars should consider differentiating the types of knowledge under exploration. Adapted rating procedure with clearer standards about the truthfulness of statements could improve psychometric quality and interpretability.

Key words. suicide, literacy, knowledge, questionnaire, instrument, psychometrics 
With an average annual mortality rate of approximately 10.7 per 100000 inhabitants worldwide (WHO, 2015), suicide was the seventeenth leading cause of death in 2015 (WHO, 2016). However, contrary to cardio-vascular diseases, diabetes or HIV/AIDS, suicide still lags behind in terms of public interest and governmental prevention efforts (Lytle et al., 2016). Yet the aftermath of suicide is devastating both in terms of human and economic costs (Shepard et al., 2016). ${ }^{\dagger}$

Literacy has been defined as a combination of health-oriented knowledge and beliefs that empower people in the recognition, prevention and management of health conditions (Jorm, 2000). It is an important building block to ground health policies in general, and mental health policies in particular. When dealing with suicide prevention, the structuring role of literacy is of special relevance. The World Health Organization (WHO) has identified multimodal policies as the main path of progress to reduce suicide-related morbi-mortality (WHO, 2014). By advocating for such an integrative approach, the WHO made clear the crucial need for better coordination between the different stakeholders. This, however, is considerably challenged by the diversification of the prevention community, with actors coming from backgrounds as varied as healthcare, associative sector, social welfare, policy making, journalism or web activism. In this context, improvement in knowledge is considered

Abbreviations: ATAS-Q = Attitude Towards Attempted Suicide-Questionnaire; ATTS = Attitudes toward suicide; BIRFFS $=$ The Beliefs in the Inheritance of Risk Factors for Suicide Scale; COSMIN = Consensus-based Standards for the selection of health status Measurement Instruments; CASQ-HK = Chinese Attitudes Toward Suicide Questionnaire-Hong Kong; EATSS = Eskin's Attitudes Toward Suicide Scale; GK = General knowledge about youth suicide/General knowledge for youth participant; KAS = Knowledge and Attitudes about Suicide; LOSS = Literacy of Suicide Scale; PCA = Principal Component factor Analysis; PFCKT = Preparing for Crisis - Knowledge Test; RFOS = Revised Facts on Suicide Quiz; SBAQ = Suicide Behaviour Attitude Questionnaire; SIQ = Suicide Intervention Questionnaire; SKT = Suicide Knowledge Test; SOQ = Suicide Opinion Questionnaire; SUIATT = Suicide Attitude Questionnaire; WHO = World Health Organization 
a major tool to empower prevention actors individually, but also, and more importantly, to provide a shared referential within which cooperation is enabled.

Beyond its background function, gain in literacy has also become the explicit goal of many universal, indicated and targeted prevention strategies. As a preferred path toward improvement of prevention skills, knowledge provision plays a key role in training programs for professional caregivers (Ryan et al., 2017), healthcare trainees (Kato et al., 2010), or gatekeepers (Cimini et al., 2014; Cross et al., 2011). Other knowledge-based prevention approaches focus on debuking myths and misconceptions that surround suicide in public opinion. By conveying correct information, education programs intend to undermine stigmatizing attitudes toward individuals concerned by suicide ideations and behaviours, alleviate help-seeking barriers, and ultimately facilitate access to care. Universal education is achieved either through direct communication campaigns (such as the Suicide Awareness Voices of Education program) (SAVE, 2019) or indirectly by working with information broadcasters. For instance, several initiatives aimed at sensitizing journalists to encourage a more responsible, cautious and informative coverage of suicide facts (O'Carroll and Potter, 1994; Ortiz and Khin Khin, 2018). More recently, measures have also been proposed to address the spread of spurious information on the internet, but also to use websites, apps, or social media to increase the web-users' knowledge of suicidal behaviours (Robinson et al., 2015).

In the past four decades, scholars have undertaken efforts in measuring literacy of suicide in a standardized, rigorous way. Some of them have developed ad-hoc assessment tools to prove the efficacy of educational or training programs (eg. Harrod et al., 2014). Others have validated scales or questionnaires before making them available for available for the scientific community (eg. Ghasemi et al., 2015; Kodaka et al., 2011). In an evidenced- 
based perspective, the possibility of rigorously accessing literacy and knowledge of suicide is a necessary condition for the implementation of prevention campaigns based on education, training or sensitization. Reliable and valid measures of literacy allow for targeting and tailoring the actions to conduct, measuring the direct impact of these actions on the participants, and assessing the global public health benefits of the program.

However, knowledge and literacy are difficult concepts to appraise. First, they refer to both objective and subjective components that can be hard to disentangle. For instance, the idea that suicide doesn't result from a rational choice is supported by clinical, neuropsychological or neuroscientific evidence (Jollant et al., 2005, 2011), but cannot be completely abstracted from the intimate convictions of individuals. Second, the concept of knowledge of suicide might not be uniform, as it can refer to contents ranging from epidemiology to phenomenology, clinical observations, neuroscientific evidence or prevention. Third, the notions of knowledge and literacy overlap with other related concepts, such as misconceptions, myths, attitude, prejudice or stigma.

This conceptual complexity translates into two interwoven methodological challenges. The first challenge is epistemological in nature, because the way a scale measures a construct greatly depends on the implicit definition that is assumed. In the cases of knowledge or literacy, the notional polysemy has complicated the stabilization of consensual definitions. Due to this unsolved conceptual blur, it is unclear whether and to what extent the constructs covered by currently available tools match. The second challenge is purely metrological. Getting a valid, reliable and sensitive measure of a construct as complex as literacy is an issue per se. When it comes to measuring them, literacy and knowledge raise the difficult question of the parsimony between internal coherence and comprehensiveness. Related scales have to find a compromise between, on the one hand, high dimensionality with strong internal 
coherence but risk of overfitting, and, on the other hand, lower dimensionality with possibly lower internal coherence, but broader comprehension of the construct.

As a first step in overcoming these methodological obstacles, we intended to draw an overview of how scholars have measured literacy of suicide so far. More precisely, the goal of the present systematic review was to address two fundamental questions :

(1) An epistemological question : what are the actual constructs measured so far under the labels "knowledge" or "literacy" of suicide?

(2) A metrological question: what is the metrological quality of the tools that have been presented or employed in the literature to measure literacy or knowledge about suicide and suicidal behaviours?

\section{Methods}

\section{Information sources and identification of records}

We conducted a systematic review of the literature in accordance with the PRISMA standards (Moher et al., 2009). Initial research was performed on the PubMed and psychINFO databases with no time restriction. The research algorithm we used to identify relevant records had to take into account the semantic overlaps mentioned in the Introduction. We thus conducted a preliminary limited scoping review to choose the keywords that would maximize the intended retrieval of instruments genuinely related to literacy or knowledge of suicide and minimize the non-intended retrieval of instruments that cover other kindred concepts. It first appeared that the terms "myth" and "misconception" were frequently used as a negative correlate of knowledge. By contrast, the word "stigma", which encompasses negative attitudes based on prejudice, but also related discriminatory behaviours (Corrigan et al., 2005), was consensually used distinctly - although relatedly - from the concept of literacy. Finally, we observed that the term "attitude" was sometimes equivocal. Its cognitive component, which includes beliefs regarding a social object (Diekstra and Kerkhof, 1988), 
could be hard to unravel from the notion of knowledge. We thus kept it as a relevant keyword. The final algorithm was as follows: "(literacy OR knowledge OR myth OR misconception OR attitud*) AND suicid*”. We monitored the databases for updates in our scope of interest until 20 april 2020. We also checked each articles' references to identify relevant papers that the algorithm might have missed.

\section{Selection of the papers}

A first screening was performed on titles and abstracts. Selection criteria were set to meet the twofold requirement of retrieving all the scales that have been proposed to measure knowledge of suicide, and of exhaustively identifying the psychometric measures that have been performed for these scales. We thus kept (a) validation studies related to an instrument designed to assess knowledge or literacy of suicide; (b) studies where authors designed, for the purpose of the evaluation, an ad-hoc instrument to assess knowledge of suicide; (c) studies where authors assessed knowledge of suicide by mean of a formerly validated or an already employed ad-hoc instrument, provided new psychometric indices were calculated on the study sample.

Non-selection criteria were: (a) records dealing with non-suicidal self-injury; (b) records dealing with assisted suicide or euthanasia; (c) records dealing with knowledge of mental illness, without any reference to suicide; (d) records dealing with stigma; (e) reviews and meta-analysis; (f) duplicates; (g) non-English records. We further screened the full text of the remaining references to exclude (a) papers for which non-compliance to the selection criteria was not clear from the title and abstract; (b) papers referring to scales for which the detailed content of some or all of the items remained unavailable, even after contacting the authors.

\section{Analysis}

\section{Psychometric properties}


For each paper, we checked whether authors assessed the following psychometric properties, and collected corresponding estimates:

- Structural validity was assessed by checking whether a Principal Component factor Analysis (PCA) was performed. In such cases, we reported whether the analysis was exploratory or confirmatory, as well as the number of identified dimensions.

- Internal consistency was examined by collecting Cronbach's $\alpha$ corresponding to the whole scale, and, when relevant, to each dimension identified from the PCA. Values above 0.70 can be considered indicative of an acceptable homogeneity

- Test-retest reliability was checked for the studies that included repeated measurements. We spotted Intraclass Correlation Coefficient (ICC) or Cohen's kappa Correlation Coefficient as informative for the stability of the overall and subscale scores over the assessment times.

- Content validity, which characterizes the extent to which an instrument reflects the construct to measure, was examined through the thematic analysis (cf. Thematic analysis section). Comparing the content of the instruments in terms of the knowledge they inquire provides an insight of their comprehensiveness and of the relevance of their items.

- Responsiveness was examined in each study that used one of the instruments to measure the effect of an educational or training program. For these articles, we reported the results of the pre-test / post-test score comparisons. When the designs comprised a control group, we rather reported, when available, the results of the Time (i.e. pre-test / post-test) x Group interaction.

- The Feasibility of using the instrument in a study or in the clinical practice was approximated by the mean completion time reported by the authors.

\section{Quality assessment}


To measure the quality of selected studies as regards to the validation procedure, we used the Consensus-based Standards for the selection of health status Measurement INstruments"' (COSMIN) checklist (MOKKINK ET AL., 2010). The COSMIN methodology consists of confronting the design and statistical methods used to test the psychometric properties of a given instrument to consensual quality standards. For each study, we rated the conformity of the methods employed to estimate psychometric indicators on a five-level scale: poor, fair, good and excellent. For each psychometric property, this global quality score was obtained by taking the lowest rating in response to a list of specific quality questions.

\section{Thematic analysis.}

We exhaustively collected the items from the included scales and questionnaires, and merged statements that were similar. To reduce redundancies and extract the core information from this extensive qualitative material, we applied two analytical steps inspired from content analysis of discourse in line with the grounded theory (STRAUSS AND CORBIN, 2006). Of note, items related to moral opinions about suicide were discarded.

As a first step, we clustered items based on the redundancies of their meaning, i.e. on the nature or type of knowledge tested. For each cluster, we smoothed formal variations of the inquiries (e.g. generality versus illustrations, negative versus positive formulations, use of synonyms etc.) by producing condensed valid utterances of the core underlying ideas. This step is akin to the labelling procedure in grounded theory.

The second step consisted in classifying the resulting summary statements by applying a thematic analysis method. Considering that each statement consisted in a semantic unit, we inferred thematic categories of meaning that could parsimoniously encompass items based on the assimilability of their sense. In grounded theory, such creation of homogeneous groups of meaning corresponds to the categorizing procedure. 


\section{Results}

\section{Study selection}

The selection flowchart is shown Figure 1. The research algorithm led to the extraction of 7,695 records. Twelve additional papers were retrieved from articles' references. After removal of 11 duplicates, and screening of titles and abstracts, we identified 216 eligible records, of which 168 were discarded after full-text examination. In total, 48 studies fulfilling selection criteria were included for the qualitative analysis.

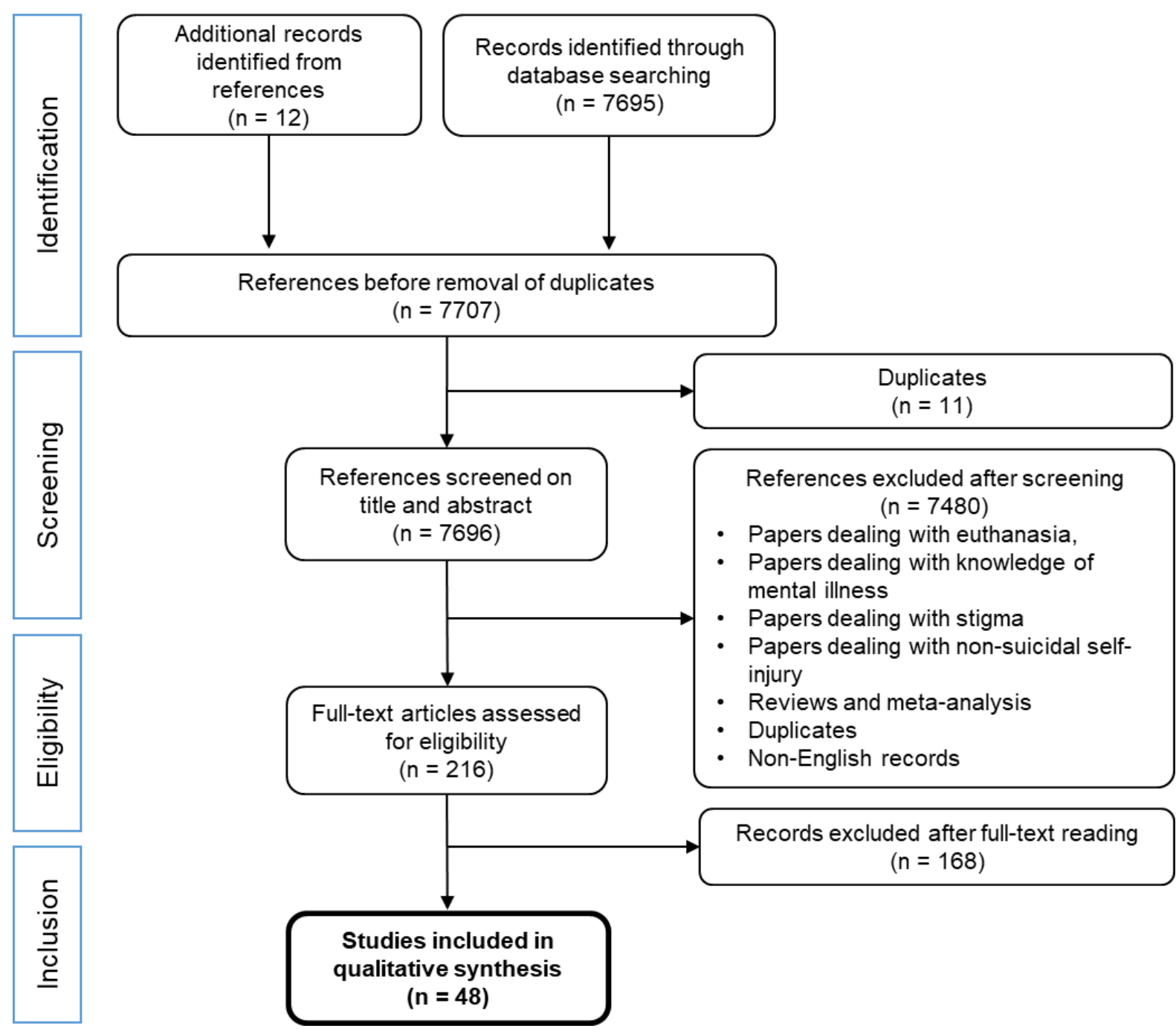

\section{Characteristics of the instruments}

Across the 48 included papers, we identified 18 unique scales assessing knowledge of suicide. Their characteristics are summarized in Table 1. 
All retrieved scales were self-administered questionnaires. Among them, 14 were formally validated, some several times (Bean and Baber, 2011; Botega et al., 2005; Diekstra and Kerkhof, 1988; Domino et al., 1982; Eskin, 1995; Hubbard and McIntosh, 1992; Kaniwa et al., 2012; Lee et al., 2007; Mackesy-Amiti et al., 1996; Ouzouni and Nakakis, 2009; Renberg and Jacobsson, 2003; Sun et al., 2007; Voracek et al., 2007a; Wallin and Runeson, 2003). Four were used as ad-hoc tools with no formal validation (Beautrais et al., 2004; Hubbard and McIntosh, 1992; Kaniwa et al., 2012; Shaffer et al., 1990). The Revised Facts on Suicide Quiz (RFOS) was initially used unvalidated in observational studies but later submitted to a validation procedure (Hubbard and McIntosh, 1992; Kõlves et al., 2007; Voracek et al., 2008a). Finally, 3 scales are referred to as validated but the corresponding results remain unpublished: the Suicide Intervention Questionnaire (SIQ) (Tierney, 1994), the Literacy of Suicide Scale (LOSS) (Chan et al., 2014) and Suicide Knowledge Test (SKT) (Spirito et al., 1988). Validation samples were mostly composed of students (eg. Anderson et al., 2008; Chan et al., 2014; Shaffer et al., 1990; Voracek et al., 2008c) or healthcare professionals (e.g. Botega et al., 2005; Domino et al., 1982; Ouzouni and Nakakis, 2009; Siau et al., 2017). For several scales, validation procedures were replicated across studies, sometimes in different languages. From that respect, most robustly validated instruments are the Suicide Opinion Questionnaire (SOQ) (Anderson et al., 2008; Beautrais et al., 2004; Domino et al., 1982, 1988, 2000; Leane and Shute, 1998; Lee, 2019; Limbacher and Domino, 1986; McAuliffe et al., 2003; Rogers and DeShon, 1992, 1995; VanSickle et al., 2016), the Attitudes toward suicide (ATTS) (Estrada et al., 2019; Ji et al., 2016; Kodaka et al., 2013; Mofidi et al., 2008; Norheim et al., 2013; Renberg and Jacobsson, 2003; Siau et al., 2017) and The Beliefs in the Inheritance of Risk Factors for Suicide Scale (BIRFFS) (Voracek et al., 2007a, 2007b, 2007c, 2008c, 2008b), which are internationally available. 
The questionnaires varied markedly in terms of number of items, with extremes ranging from 6 (e.g. the General knowledge about youth suicide/General knowledge for youth participant - GK) (Bean and Baber, 2011) to 107 (e.g. the SOQ) (Domino et al., 1982), but mostly comprised 20 to 40. Likewise, there were high between and within-scale discrepancies in the number of dimensions revealed by the exploratory PCAs. Highest dimensionality was found for the Suicide Attitude Questionnaire (SUIATT) with 19 components (Diekstra and Kerkhof, 1988).

When tested, global internal consistencies generally revealed acceptable (i.e. $\alpha \geq 0.7$ ), except for the RFOS, Kaniwas et al.'s scale and the Preparing for Crisis - Knowledge Test (PFC-KT) for which Cronbach's $\alpha$ were found questionable or poor (i.e. < 0.5) (Hubbard and McIntosh, 1992; Kaniwa et al., 2012; Mackesy-Amiti et al., 1996). By contrast, Vansickle et al. (2016), Diekstra \& Kerkhof (1988), and Ouzouni \& Nakakis (Ouzouni and Nakakis, 2009) found excellent internal consistencies (i.e. $\alpha \geq 0.9$ ) for the SOQ, the SUIATT and the Attitude Towards Attempted Suicide-Questionnaire (ATAS-Q), respectively. As regards the internal consistency of the scales' dimensions, $\alpha$ values showed marked variations depending on the validation sample, with the robust observation that the higher the dimensionality, the higher the number of components with poor consistency.

Test-retest reliability was checked only in 6 instruments: the SOQ (Domino et al., 1988, 2000; Lee, 2019; McAuliffe et al., 2003; Rogers and DeShon, 1995), the SIQ (Tierney, 1994), the SUIATT (Diekstra and Kerkhof, 1988), the ATTS (Ji et al., 2016), the PFC-KT (Mackesy-Amiti et al., 1996) and the BIRFFS (Voracek et al., 2007a, 2007b). Reported correlations were all significant with generally strong size effects $(r>0.7)$, except for the PFC-KT for which it was moderate $(r=0.36)$.

Responsiveness was tested in only a few scales: scores at the GK, Suicide Behaviour Attitude Questionnaire (SBAQ) and PFC-KT significantly increased in participants of 
educational or training programs (Bean and Baber, 2011; Botega et al., 2007; La Guardia et al., 2019; Mackesy-Amiti et al., 1996); similarly, studies that used the SIQ or the SKT to measure the pre-test / post-test difference in participants who received a workshop or awareness program as compared to a control group evidenced significant interactions between the effect of assessment time and the effect of group (Spirito et al., 1988; Tierney, 1994).

Only the BIRFFS received validation from its external coherence, as Voracek et al. (Voracek et al., 2007a, 2007b, 2007c, 2008b, 2008b) tested the correlation of its score with this of the RFOS. Results were significant but $r$ values revealed weak to moderate.

Although authors rarely addressed feasibility, we know it takes 30 minutes to complete the SOQ (Domino et al., 1982, 1988, Rogers and DeShon, 1992, 1995), less than 30 minutes to complete the BIRFFS (Voracek et al., 2007a, 2007b, 2007c), and 15 minutes to complete the PFC-KT (Mackesy-Amiti et al., 1996). 
Table 1. Characteristics and psychometric properties of selected scales inquiring knowledge of suicide, as assessed by corresponding validation studies.

\begin{tabular}{|c|c|c|c|c|c|}
\hline Scale & Author & Country & Sample & $\mathrm{N}$ Items & Psychometric properties \\
\hline \multirow[t]{6}{*}{$\begin{array}{l}\text { Suicide Opinion } \\
\text { Questionnaire (SOQ) }\end{array}$} & $\begin{array}{l}\text { Domino et } \\
\text { al., } 1982\end{array}$ & USA & $\begin{array}{l}\text { Medical doctors and PhD students } \\
(\mathrm{N}=285) \\
\text { - Female: } 51 \% \\
\text { - Median age }=36 \text { (range: } 23-71)\end{array}$ & $100+7$ & $\begin{array}{l}\text { D. } 15 \text { dimensions (exploratory PCA) } \\
\text { IC. } \alpha=0.41,0.76,0.67,0.75,0.31,0.14,0.13,0.08,0.42 \text {, } \\
0.29,0.19,0.04,0.54,0.46,0.20 \\
\text { F. } 30 \text { min }\end{array}$ \\
\hline & $\begin{array}{l}\text { Domino et } \\
\text { al., } 1988\end{array}$ & $\begin{array}{l}\text { USA and } \\
\text { New- } \\
\text { Zealand }\end{array}$ & $\begin{array}{l}\text { Students }(\mathrm{N}=484) \\
\text { - Female: } 65 \% \\
\text { - Age range }=18-21\end{array}$ & $100+7$ & $\begin{array}{l}\text { D. } 8 \text { dimensions (confirmatory PCA) } \\
\text { R. Test-retest: } r=0.83,0.86,0.79,0.82,0.76,0.77,0.75 \text {, } \\
0.75 \\
\text { F. } 30 \mathrm{~min}\end{array}$ \\
\hline & $\begin{array}{l}\text { Limbacher \& } \\
\text { Domino, } \\
1985-86\end{array}$ & USA & $\begin{array}{l}\text { Students }(\mathrm{N}=649) \\
\text { - Female: } 64 \% \\
\text { - Age: NA }\end{array}$ & $100+7$ & D. 7 dimensions (confirmatory PCA) \\
\hline & $\begin{array}{l}\text { Rogers et al., } \\
1992\end{array}$ & USA & $\begin{array}{l}\text { Students }(\mathrm{N}=237) \\
\text { - Female: } 60 \% \\
\text { - Mean age }=20.9(\mathrm{SD} \pm 5.3)\end{array}$ & 100 & $\begin{array}{l}\text { D. } 5 \text { dimensions (exploratory and confirmatory PCA) } \\
\text { IC. } \alpha=0.88,0.74,0.72,0.73,0.59 \\
\text { F. } 30 \text { min }\end{array}$ \\
\hline & $\begin{array}{l}\text { Rogers et al., } \\
1995\end{array}$ & USA & $\begin{array}{l}\text { Students }(\mathrm{N}=452) \\
\text { - Female: } 61 \% \\
\text { - Mean age }=23.0(\mathrm{SD} \pm 7.0)\end{array}$ & $52+3$ & $\begin{array}{l}\text { D. } 6 \text { dimensions (confirmatory PCA) } \\
\text { IC. } \alpha=0.85,0.66,0.48,0.55,0.68,0.59 \\
\text { R. Test-retest } r=0.92,0.78,0.55,0.77,0.34,0.51 \\
\text { F. } 30 \text { min }\end{array}$ \\
\hline & $\begin{array}{l}\text { Leane \& } \\
\text { Shute, } 1998\end{array}$ & Australia & $\begin{array}{l}\text { Teachers and clergy members }(\mathrm{N}=219) \\
\text { - Female: } 35 \% \\
\text { - Median age }=43 \text { (range: } 23-67)\end{array}$ & 52 & $\begin{array}{l}\text { D. } 5 \text { dimensions (confirmatory PCA) } \\
\text { IC. } \alpha=0.88,0.67,0.65,0.55,0.44\end{array}$ \\
\hline
\end{tabular}




\begin{tabular}{|c|c|c|c|c|c|}
\hline & $\begin{array}{l}\text { Domino et } \\
\text { al., } 2000\end{array}$ & $\begin{array}{l}\text { USA and } \\
\text { Taiwan }\end{array}$ & $\begin{array}{l}\text { USA } \\
\text { Students and adults }(\mathrm{N}=218) \\
- \text { Female: } 45 \% \\
\text { - Median age }=\mathrm{NA} \\
\text { Taiwan } \\
\text { Adults }(\mathrm{N}=291) \\
\text { - Female: NA } \\
\text { - Median age }=42(\text { range }=17-55)\end{array}$ & $100+7$ & $\begin{array}{l}\text { D. SOQ-C [Content Scale of SOQ developed by Domino, } \\
\text { MacGregor, and Hannah (1988-89)]: } 8 \text { dimensions; SOQ-F } \\
\text { [Factor Analytic Scale of the SOQ developed by Rogers and } \\
\text { DeShon (1993)]: } 5 \text { dimensions (confirmatory PCA for both) } \\
\text { IC. USA. SOQ-C: } \alpha=0.68,0.71,0.53,0.54,0.58,0.67 \text {, } \\
\text { 0.51, 0.62; SOQ-F: } \alpha=0.78,0.71,0.74,0.69,0.52 ; \text { Taiwan. } \\
\text { SOQ-C: } \alpha=0.72,0.76,0.63,0.51,0.56,0.71,0.63,0.64 \text {; } \\
\text { SOQ-F: } \alpha=0.83,0.73,0.68,0.71,0.52 \\
\text { R. USA. Test-retest SOQ-C: } r=0.87,0.85,0.83,0.87,0.85 \text {, } \\
\text { 0.77, } 0.77,0.75 ; \text { SOQ-F: } r=0.86,0.82,0.79,0.870 .74 \\
\text { Taiwan: NA }\end{array}$ \\
\hline & $\begin{array}{l}\text { McAuliffe et } \\
\text { al., } 2003\end{array}$ & Ireland & $\begin{array}{l}\text { Students }(\mathrm{N}=328) \\
\text { - Female: } 49 \% \\
\text { - Mean age }=19.6(\mathrm{SD} \pm 2.2)\end{array}$ & 40 & $\begin{array}{l}\text { D. } 4 \text { dimensions (a priori defined, not tested) } \\
\text { IC. } \alpha=0.56,0.52,0.73,0.41 \\
\text { R. Test-retest: } 0.67,0.65,0.76,0.58(p<0.001)\end{array}$ \\
\hline & $\begin{array}{l}\text { Beautrais et } \\
\text { al., } 2004\end{array}$ & $\begin{array}{l}\text { New } \\
\text { Zealand }\end{array}$ & - & 3 & - \\
\hline & $\begin{array}{l}\text { Anderson et } \\
\text { al., } 2008\end{array}$ & USA & $\begin{array}{l}\text { Master students and general population } \\
(\mathrm{N}=568) \\
\text { - Female: } 72 \% \\
\text { - Mean age }=43.0(\mathrm{SD} \pm 15.2)\end{array}$ & $100+7$ & $\begin{array}{l}\text { D. } 2 \text { dimensions (exploratory and confirmatory PCA) } \\
\text { IC. } \alpha=0.86,0.86\end{array}$ \\
\hline & & & $\begin{array}{l}\text { Students in social science }(\mathrm{N}=288) \\
\text { - Female: } 75 \% \\
\text { - Mean age }=22.5(\mathrm{SD} \pm 5.3)\end{array}$ & 32 & $\begin{array}{l}\text { D. Models with } 15,8,5 \text { and } 2 \text { dimensions structures failed } \\
\text { to account for the observed data }\end{array}$ \\
\hline & $\begin{array}{l}\text { Vansickle et } \\
\text { al., } 2016\end{array}$ & USA & $\begin{array}{l}\text { Soldiers }(\mathrm{N}=1,758) \\
\text { - Female: } 8 \% \\
\text { - Age: NA }\end{array}$ & 107 & $\begin{array}{l}\text { D. } 5 \text { dimensions (confirmatory PCA) } \\
\text { IC. } \alpha=0.93,0.83,0.54,0.68,<0.40 \text {; global } \alpha=0.94\end{array}$ \\
\hline & Lee, 2019 & $\begin{array}{l}\text { South } \\
\text { Korea }\end{array}$ & Undergraduates $(\mathrm{N}=195)$ & 66 & $\begin{array}{l}\text { D. } 12 \text { dimensions (confirmatory PCA) } \\
\text { IC. Eight had Cronbach's } \alpha \geq 0.7 \\
\text { R. Four had ICC } \geq 0.6\end{array}$ \\
\hline $\begin{array}{l}\text { Suicide } \\
\text { Intervention } \\
\text { Questionnaire (SIQ) }\end{array}$ & $\begin{array}{l}\text { Tierney, } \\
1988, \\
\text { unpublished, } \\
\text { reported in } \\
\text { Tierney, } 1994\end{array}$ & Canada & NA & 20 & $\begin{array}{l}\text { R. Test-retest: } r=0.82 \text {; split-half: } r=0.79 \\
\text { Rs. Administration before (pre-test) and after (post-test) a } \\
\text { workshop (intervention group) vs at } 2 \text { days interval without } \\
\text { intervention (control group) } \\
\text { Group x Time interaction (ANOVA): } F=39.36, p<.001\end{array}$ \\
\hline
\end{tabular}




\begin{tabular}{|c|c|c|c|c|c|}
\hline $\begin{array}{l}\text { Suicide Knowledge } \\
\text { Test (SKT) }\end{array}$ & $\begin{array}{l}\text { Spirito et al., } \\
1988\end{array}$ & USA & - & 21 & $\begin{array}{l}\text { Rs. Administration before (pre-test) and after (post-test) a } \\
\text { suicide awareness program (intervention group) vs at } 2 \\
\text { different timepoints in a similar timeframe interval without } \\
\text { intervention (control group) } \\
\text { Group x Time interaction (ANOVA): Hotelling's } t=1.81 \text {, } \\
p<0.030\end{array}$ \\
\hline $\begin{array}{l}\text { Suicide Attitude } \\
\text { Questionnaire } \\
\text { (SUIATT) }\end{array}$ & $\begin{array}{l}\text { Diekstra \& } \\
\text { Kerkhof, } \\
1988\end{array}$ & $\begin{array}{l}\text { Nether- } \\
\text { lands }\end{array}$ & $\begin{array}{l}\text { Adults and young adults }(\mathrm{N}=712) \\
\text { - Female: } 57 \% \\
\text { - Mean age }=43.6 \text { (range: } 15-68)\end{array}$ & 63 & $\begin{array}{l}\text { D. } 19 \text { dimensions (exploratory and confirmatory PCA) } \\
\text { IC. } \alpha=0.42,0.33,0.36,0.50,0.52,0.72,0.45,0.39,0.36, \\
0.82,0.83,0.72,0.75,0.82,0.82(\alpha=\text { NA for } 4 \text { dimensions); } \\
\text { global } \alpha=0.90 \\
\text { R. } r=0.82(p=.020)\end{array}$ \\
\hline $\begin{array}{l}\text { Knowledge and } \\
\text { Attitudes about } \\
\text { Suicide (KAS) }\end{array}$ & $\begin{array}{l}\text { Shaffer et al., } \\
1990\end{array}$ & USA & $\begin{array}{l}\text { Students }(\mathrm{N}=524) \\
\text { - Female: } 54 \% \\
\text { - Mean age }=14.2(\mathrm{SD} \pm \mathrm{NA})\end{array}$ & 48 & $\begin{array}{l}\text { R. Cross-examiner: } \kappa=0.22-0.51 \\
\text { Rs. Administration before (pre-test) and } 1 \text { month after (post- } \\
\text { test) a school-based suicide prevention program } \\
\text { (intervention group) vs at } 2 \text { different timepoints in a similar } \\
\text { timeframe interval without intervention (control group) } \\
\text { No statistical test available }\end{array}$ \\
\hline \multirow[t]{3}{*}{$\begin{array}{l}\text { Revised Facts on } \\
\text { Suicide Quiz (RFOS) }\end{array}$} & $\begin{array}{l}\text { Hubbard \& } \\
\text { McIntosh, } \\
1992 \\
\end{array}$ & USA & Students $(\mathrm{N}=331)$ & 36 & - \\
\hline & $\begin{array}{l}\text { Kolves et al., } \\
2007\end{array}$ & Estonia & $\begin{array}{l}\text { Students in psychology and sociology } \\
(\mathrm{N}=107) \\
\text { - Female: } 86 \% \\
\text { - Mean age }=23.5(\mathrm{SD} \pm 3.1)\end{array}$ & 34 & $\begin{array}{l}\text { D. } 2 \text { parts (a priori defined, not tested) } \\
\text { IC. } \alpha=0.59,0.47 ; \text { global } \alpha=0.67\end{array}$ \\
\hline & $\begin{array}{l}\text { Voracek et } \\
\text { al., 2008a }\end{array}$ & Austria & $\begin{array}{l}\text { Medical students and psychology } \\
\text { students }(\mathrm{N}=1,093) \\
\text { - Female: } 65 \% \\
\text { - Mean age }=25.0(\mathrm{SD} \pm 6.6)\end{array}$ & 34 & $\begin{array}{l}\text { D. } 2 \text { dimensions (confirmatory PCA) } \\
\text { IC. } \alpha=0.59,0.48\end{array}$ \\
\hline $\begin{array}{l}\text { Eskin's Attitudes } \\
\text { Toward Suicide } \\
\text { Scale (EATSS) }\end{array}$ & Eskin, 1995 & Sweden & $\begin{array}{l}\text { Swedish High School student }(\mathrm{N}=167) \\
\text { - Female: } 58 \% \\
\text { - Mean age }=18.0 \text { yo }(\mathrm{SD} \pm 1.0)\end{array}$ & 20 & IC. $\alpha=0.70$ \\
\hline
\end{tabular}




\begin{tabular}{|c|c|c|c|c|c|}
\hline & \multirow{2}{*}{\multicolumn{2}{|c|}{ Eskin, $2004 \quad$ Turkey }} & \multirow[b]{2}{*}{$\begin{array}{l}\text { Turkish high school students with secular } \\
\text { education }(\mathrm{N}=214) \\
\text { - Female: } 41 \% \\
\text { - Mean age }=17.0(\mathrm{SD} \pm 1.0) \\
\text { High school students with religious } \\
\text { education }(\mathrm{N}=206) \\
\text { - Female: } 50 \% \\
\text { - Mean age }=16.4(\mathrm{SD} \pm 1.2)\end{array}$} & \multirow[b]{2}{*}{24} & \multirow[b]{2}{*}{$\begin{array}{l}\text { D. } 6 \text { dimensions (confirmatory PCA) } \\
\text { IC. NA }\end{array}$} \\
\hline & & & & & \\
\hline & $\begin{array}{l}\text { Nader et al., } \\
2012\end{array}$ & Germany & $\begin{array}{l}571 \text { volunteer adults } \\
\text { - Female: } 63 \% \\
\text { - Mean age }=30.0(\mathrm{SD} \pm 12.8) \\
\end{array}$ & 24 & $\begin{array}{l}\text { D. } 6 \text { dimensions (confirmatory PCA) } \\
\text { IC. } \alpha=0.89,0.92,0.93,0.84,0.90,0.42\end{array}$ \\
\hline $\begin{array}{l}\text { Preparing for Crisis - } \\
\text { Knowledge Test } \\
\text { (PFC-KT), A and B } \\
\text { forms }\end{array}$ & $\begin{array}{l}\text { Mackesy- } \\
\text { Amiti et al., } \\
1996\end{array}$ & USA & $\begin{array}{l}\text { School Staff and general population } \\
(\mathrm{N}=205) \\
\text { - Female: } 70 \% \\
\text { - Age: NA }\end{array}$ & $\begin{array}{l}25 \\
\text { for each } \\
\text { form }\end{array}$ & $\begin{array}{l}\text { D. Form A: } 6 \text { dimensions; form B: } 8 \text { dimensions } \\
\text { (confirmatory PCA) } \\
\text { IC. Form A: global } \alpha=0.58 \text {; form B: global } \alpha=0.52 \\
\text { R. Test-retest: } r=0.36(p<0.001) \\
\text { Rs. Administration before (pre-test) and after (post-test) a } \\
\text { proactive training program for suicide postvention } \\
\text { Pre-test/post-test comparison: Student's } t=11.37, p<.001 \\
\text { F. } 15 \text { min }\end{array}$ \\
\hline \multirow[t]{5}{*}{$\begin{array}{l}\text { Attitudes toward } \\
\text { suicide (ATTS) }\end{array}$} & \multirow[t]{2}{*}{$\begin{array}{l}\text { Renberg \& } \\
\text { Jacobsson, } \\
2003\end{array}$} & \multirow[t]{2}{*}{ Sweden } & $\begin{array}{l}\text { Adults }(\mathrm{N}=510) \\
\text { - Female: } 56 \% \\
\text { - Age range }=18-65\end{array}$ & 20 & $\begin{array}{l}\text { D. } 8 \text { dimensions (exploratory PCA) } \\
\text { IC. } \alpha=0.66,0.60,0.40,0.65,0.34,0.53,0.45,0.38 \text {; global } \\
\alpha=0.51\end{array}$ \\
\hline & & & $\begin{array}{l}\text { Adults }(\mathrm{N}=640) \\
\text { - Female: } \mathrm{NA} \\
\text { - Age range }=18-65\end{array}$ & 34 & $\begin{array}{l}\text { D. } 10 \text { dimensions (exploratory and confirmatory PCA) } \\
\text { IC. } \alpha=0.86,0.72,0.64,0.63,0.45,0.51,0.51,0.45,0.38 \text {, } \\
0.54,0.60 \text {; global } \alpha=0.60\end{array}$ \\
\hline & $\begin{array}{l}\text { Mofidi et al, } \\
2008\end{array}$ & Iran & $\begin{array}{l}\text { Adults }(\mathrm{N}=996) \\
\text { - Female: } 52 \% \\
\text { - Mean age }=\mathrm{NA}\end{array}$ & 34 & $\begin{array}{l}\text { D. } 3 \text { dimensions (exploratory and confirmatory PCA) } \\
\text { IC. } \alpha=0.76,0.60,0.54 \text {; global } \alpha=0.65\end{array}$ \\
\hline & $\begin{array}{l}\text { Kodaka et al, } \\
2013\end{array}$ & Japan & $\begin{array}{l}\text { Adults }(\mathrm{N}=842) \\
\text { - Female: } 70 \% \\
\text { - Mean age }=44.1(\mathrm{SD} \pm 11.4)\end{array}$ & 37 & $\begin{array}{l}\text { D. } 6 \text { dimensions (exploratory PCA) } \\
\text { IC. } \alpha=0.75,0.63,0.65,0.61,0.44,0.34\end{array}$ \\
\hline & $\begin{array}{l}\text { Norheim et } \\
\text { al, } 2013\end{array}$ & Norway & $\begin{array}{l}\text { Medical staff }(\mathrm{N}=226) \\
\text { - Female: } 65 \% \\
\text { - Age range }=30-50\end{array}$ & 37 & $\begin{array}{l}\text { D. } 2 \text { dimensions (confirmatory PCA) } \\
\text { IC. } \alpha=0.84,0.66\end{array}$ \\
\hline
\end{tabular}









\begin{tabular}{|c|c|c|c|c|c|}
\hline & $\begin{array}{l}\text { Sun et al., } \\
2011\end{array}$ & Taiwan & $\begin{array}{l}\text { Nurse students }(\mathrm{N}=174) \\
\text { - Female: } 98 \% \\
\text { - Mean age }=26.8(\mathrm{SD} \pm 4.7)\end{array}$ & 30 & $\begin{array}{l}\text { D. } 5 \text { dimensions (arbitrarily defined) } \\
\text { IC. } \alpha=0.33,0.47,0.67,0.61,0.54 \text {; global } \alpha=0.83 \\
\text { Rs. Administration before (pre-test) and after (post-test) a } \\
\text { suicide education program for nurses (intervention group) vs } \\
\text { before and after a routine psychiatric course (control group) } \\
\text { Post-test inter-group comparisons: } \\
\text { - Acceptability: Student's } t=-6.60, p<.001 \\
\text { - Morality and mental illness: Student's } t=-5.73, p<.001 \\
\text { - Professional role, work and care: Student's } t=-3.57 \text {, } \\
p<.001 \\
\text { - Communication and attention: Student's } t=-5.30, p<.001 \\
\text { - Beliefs: Student's } t=-5.85, p<.001\end{array}$ \\
\hline & $\begin{array}{l}\text { Siau et al., } \\
2019\end{array}$ & Malaysian & $\begin{array}{l}\text { Nurses }(\mathrm{N}=189) \\
\text { - Female: } 94 \% \\
\text { - Mean age }=32.5(\mathrm{SD} \pm 11.0) \\
\end{array}$ & 22 & IC. Global $\alpha=0.72$ \\
\hline $\begin{array}{l}\text { Chinese Attitudes } \\
\text { Toward Suicide } \\
\text { Questionnaire-Hong } \\
\text { Kong (CASQ-HK), } \\
\text { Part A }\end{array}$ & $\begin{array}{l}\text { Lee et al., } \\
2007\end{array}$ & $\begin{array}{l}\text { Hong- } \\
\text { Kong }\end{array}$ & $\begin{array}{l}\text { General population }(\mathrm{N}=1197) \\
\text { - Female: } 44 \% \\
\text { - Mean age }=\text { NA }\end{array}$ & 73 & $\begin{array}{l}\text { D. } 9 \text { dimensions (confirmatory PCA) } \\
\text { IC. } \alpha=0.39,0.74,0.69,0.57,0.62,0.60,0.51,0.33,0.31 \text {; } \\
\text { global } \alpha=0.85\end{array}$ \\
\hline $\begin{array}{l}\text { The Beliefs in the } \\
\text { Inheritance of Risk } \\
\text { Factors } \\
\text { for Suicide Scale } \\
\text { (BIRFFS) }\end{array}$ & $\begin{array}{l}\text { Voracek et } \\
\text { al., 2007a }\end{array}$ & Austria & $\begin{array}{l}\text { Psychology students }(\mathrm{N}=165) \\
\text { - Female: } 84 \% \\
\text { - Mean age }=22.3(\mathrm{SD} \pm 5.0) \\
\text { Medical students }(\mathrm{N}=70) \\
\text { - Female: } 69 \% \\
\text { - Mean age }=28.7(\mathrm{SD} \pm 7.0)\end{array}$ & 22 & $\begin{array}{l}\text { D. Psychology students sample: } 8 \text { dimensions (exploratory } \\
\text { PCA); medical students sample: } 9 \text { dimensions (exploratory } \\
\text { PCA) } \\
\text { IC. Psychology students sample: global } \alpha=0.66 \text { and } 0.68 \\
\text { (first and second testing); medical students sample: } \\
\text { global } \alpha=0.75 \\
\text { EC. Psychology students sample: Correlation with the } \\
\text { RFOS total score (Hubbard \& McIntosh, 1992): } r=0.38 \\
\text { ( } p<.001) ; \text { correlation with the } 2^{\text {nd }} \text { item of the RFOS } \\
\text { (Hubbard \& McIntosh, } 1992): r=0.41(p<.001) ; \text { medical } \\
\text { students sample: } r=0.17 \text { (NS) and } r=0.27(p<.020) \\
\text { respectively } \\
\text { R. Test-retest: } r=0.55(p<.001) \\
\text { F. }<30 \text { min }\end{array}$ \\
\hline
\end{tabular}




\begin{tabular}{|c|c|c|c|c|}
\hline $\begin{array}{l}\text { Voracek et al, } \\
2007 b\end{array}$ & Austria & $\begin{array}{l}\text { Psychology students }(\mathrm{N}=155) \\
\text { - Female: } 73 \% \\
\text { - Mean age }=21.9(\mathrm{SD} \pm 4.3)\end{array}$ & 22 & $\begin{array}{l}\text { D. } 6 \text { dimensions (exploratory PCA) } \\
\text { IC. Global } \alpha=0.74 \text { (first testing, T1) and } 0.76 \text { (retest, T2) } \\
\text { EC. Correlation with the Belief in Genetic Determinism } \\
\text { Scale (Keller, 2005): } r=0.53(p<.001) \text {; correlation with the } \\
\text { GenEthics Questionnaire (Illes et al., 2002): } r=0.21 \\
(p=.020) \\
\text { R. Test-retest } r=0.70(p<.001) \\
\text { F. }<30 \text { min }\end{array}$ \\
\hline $\begin{array}{l}\text { Voracek et } \\
\text { al., 2007c }\end{array}$ & Austria & $\begin{array}{l}\text { General population }(\mathrm{N}=159) \\
\text { - Female: } 66 \% \\
\text { - Mean age }=31.7(\mathrm{SD} \pm 12.6)\end{array}$ & 22 & $\begin{array}{l}\text { D. } 8 \text { dimensions (exploratory PCA) } \\
\text { IC. Global } \alpha=0.68 \\
\text { EC. Correlation with the } 2^{\text {nd }} \text { item of the RFOS (Hubbard \& } \\
\text { McIntosh, 1992): } r=0.43(p<.001) \\
\text { F. }<30 \text { min }\end{array}$ \\
\hline $\begin{array}{l}\text { Voracek et al, } \\
2008 b\end{array}$ & Canada & $\begin{array}{l}\text { Students }(\mathrm{N}=288) \\
\text { - Female: } 71 \% \\
\text { - Mean age }=21.3(\mathrm{SD} \pm 3.6)\end{array}$ & 22 & $\begin{array}{l}\text { D. } 7 \text { dimensions (exploratory PCA) } \\
\text { IC. Global } \alpha=0.52 \\
\text { EC. Correlation with the RFOS: } r=0.24(p<.001) \text {; } \\
\text { correlation with the } 2^{\text {nd }} \text { item of the RFOS: } r=0.40 \\
(p<.001) \text {; correlation with Belief in Genetic Determinism } \\
\text { Scale: } r=0.37(p=0.005)\end{array}$ \\
\hline $\begin{array}{l}\text { Voracek et } \\
\text { al., 2008c }\end{array}$ & $\begin{array}{l}\text { Estonia, } \\
\text { Malaysia, } \\
\text { Romania, } \\
\text { UK and } \\
\text { USA }\end{array}$ & $\begin{array}{l}\text { Students in psychology and in medicine } \\
(\mathrm{N}=686) \\
\text { - Female: } 77 \% \\
\text { - Mean age }=22.5(\mathrm{SD} \pm 5.5)\end{array}$ & 22 & $\begin{array}{l}\text { D. } 8 \text { dimensions (PCA exploratory) } \\
\text { IC. } \alpha=0.50 \\
\text { EC. Correlation with the RFOS: } r=0.17(p<0.050) \text { and } \\
r=0.30(p<0.010) \text { in the Romanian and UK samples } \\
\text { respectively; correlation with the } 2^{\text {nd }} \text { item of the RFOS: } r= \\
0.49(p<.001), 0.40(p<.001), 0.46(p<.001), 0.51 \\
(p<0.001), 0.22(p<0.050) \text { in the Estonian, Malaysian, } \\
\text { Romanian and UK samples respectively; correlation with the } \\
\text { Belief in Genetic Determinism_Scale: } r=0.49,0.40,0.37 \text {, } \\
0.45 \text { ( } p<0.001) \text { in the Estonian, Malaysian, Romanian and } \\
\text { UK samples respectively; correlation with GenEthics } \\
\text { Questionnaire: } r=0.34(p<.001) \text { in the UK sample }\end{array}$ \\
\hline $\begin{array}{l}\text { Ouzouni \& } \\
\text { Nakakis, } \\
2009\end{array}$ & Greece & $\begin{array}{l}\text { Doctors and nurses }(\mathrm{n}=186) \\
\text { - Female: } 64 \% \\
\text { - Median age }=33 \text { (range: } 24-56)\end{array}$ & 80 & $\begin{array}{l}\text { D. } 8 \text { dimensions (exploratory and confirmatory PCA) } \\
\text { IC. } \alpha=0.95,0.88,0.92,0.79,0.75,0.59,0.83,0.65 \text {; global } \\
\alpha=0.96 \\
\text { R. Test-retest: } r=0.99\end{array}$ \\
\hline
\end{tabular}




\begin{tabular}{|c|c|c|c|c|c|}
\hline $\begin{array}{l}\text { General knowledge } \\
\text { about youth suicide } \\
\text { (adult and youth } \\
\text { versions) }\end{array}$ & $\begin{array}{l}\text { Bean \& } \\
\text { Baber, } 2011\end{array}$ & USA & $\begin{array}{l}\text { Adults }(\mathrm{N}=648) \\
\text { - Female: NA } \\
\text { - Mean age }=\text { NA } \\
\text { High school students }(\mathrm{N}=204) \\
\text { - Female: NA } \\
\text { - Mean age = NA }\end{array}$ & 6 & $\begin{array}{l}\text { D. } 5 \text { dimensions (exploratory PCA) } \\
\text { IC. Global } \alpha=0.74(0.85 \text { for the adult version, } 0.74 \text { for the } \\
\text { youth version) } \\
\text { Rs. Administration before (pre-test) and after (post-test) a } \\
\text { community-based youth gatekeeper training program and } \\
\text { discipline-specific training for professional (intervention } \\
\text { groups) } \\
\text { Pre-test/post-test comparisons in professionals: } \\
\text { Students' } t=-37.80, p<.001 \\
\text { Pre-test/post-test comparisons in youth: Students' } t=-14.36 \text {, } \\
p<.001\end{array}$ \\
\hline- & $\begin{array}{l}\text { Kaniwa et al., } \\
2012\end{array}$ & Japan & $\begin{array}{l}\text { Government agents, health and } \\
\text { unspecified professionals }(\mathrm{N}=646) \\
\text { - Female: } 66 \% \\
\text { - Mean age }=42.4(\mathrm{SD} \pm 12.3)\end{array}$ & 10 & $\begin{array}{l}\text { IC. } \alpha=0.63 \\
\text { Rs. Administration before (pre-test) and after (post-test) a } \\
\text { suicide prevention education program for government } \\
\text { officers, and healthcare and welfare professionals } \\
\text { (intervention group) } \\
\text { Significant pre-test/post-test improvement of all questions' } \\
\text { scores, except in question } 1\end{array}$ \\
\hline \multirow[t]{2}{*}{$\begin{array}{l}\text { Literacy of Suicide } \\
\text { Scale (LOSS) }\end{array}$} & $\begin{array}{l}\text { Chan et al., } \\
2014\end{array}$ & Australia & $\begin{array}{l}\text { Students }(\mathrm{N}=165) \\
\text { - Female: } 55 \% \\
\text { - Median age }=25.0(\text { range }=19.0-42.0)\end{array}$ & 27 & IC. $\alpha=0.71$ \\
\hline & $\begin{array}{l}\text { Rivera- } \\
\text { Segarra et al, } \\
2018\end{array}$ & USA & $\begin{array}{l}\text { Medical students }(\mathrm{N}=123) \\
\text { - Female: } 56 \% \\
\text { - Mean age }=\text { NA }\end{array}$ & 27 & IC. $\alpha=0.83$ \\
\hline
\end{tabular}

Note. Scales' label or psychometric properties are reported only when made available or tested in the source articles.

$\alpha=$ Cronbach's alpha; BHS = Beck Hopelessness Scale; D = Dimensionality; EC = External Consistency; F = Feasibility (duration of completion in minutes); IC = Internal

Consistency; $\kappa=$ Cohen's kappa inter-rater reliability coefficient; NA = Not Acknowledged; $r=$ Intra-class or test-retest Spearman's or Pearson's correction coefficient; R =

Reliability; Rs = Responsiveness; SD = Standard Deviation; V = Validity

For each scale, the psychometric properties are specified when they have been completed. For SOQ, seven items that request demographic information. 


\section{Quality of the validation procedures}

Evaluation of the methodological quality of the validation procedures according to the COSMIN standards are summarized Table 2. In most papers, assessments of consistency, reliability and validity were rated poor or fair. Remarkable exceptions were the ATTS, the SBAQ, and the Chinese Attitudes Toward Suicide Questionnaire-Hong Kong (CASQ-HK) for which the internal consistency, the structural validity and the content validity were all at least once assessed with good or excellent methodology (Botega et al., 2005; Estrada et al., 2019; Lee et al., 2007; Renberg and Jacobsson, 2003; Siau et al., 2017). We also rated Vansickle et al.'s assessment of the structural validity of SOQ (VanSickle et al., 2016) as excellent. Reliability assessment was conducted according to good standards only for the ATTS. Among the few instruments for which the evaluation of hypothesis testing was carried out, only the SOQ and the PFC-KT complied to good methods (Anderson et al., 2008; Beautrais et al., 2004; Domino et al., 1982, 1988, 2000; Leane and Shute, 1998; Lee, 2019; Limbacher and Domino, 1986; Mackesy-Amiti et al., 1996; McAuliffe et al., 2003; Rogers and DeShon, 1992, 1995; VanSickle et al., 2016). As regards responsiveness, quality reached the good score in Mackesy-Amiti et al.'s assessment of the PFC-KT (Mackesy-Amiti et al., 1996), in Botega et al.'s assessment of the SBAQ (Botega et al., 2007), in Bean \& Baber's assessment of the GK (Bean and Baber, 2011) and Kaniwa et al.'s scale (Kaniwa et al., 2012). 
Table 2. Quality of validation studies per measurement property and questionnaire

\begin{tabular}{|c|c|c|c|c|c|c|c|c|}
\hline & $\begin{array}{c}\text { Internal } \\
\text { consistency }\end{array}$ & Reliability & $\begin{array}{l}\text { Content } \\
\text { validity }\end{array}$ & $\begin{array}{c}\text { Structural } \\
\text { validity }\end{array}$ & $\begin{array}{l}\text { Hypothesis } \\
\text { testing }\end{array}$ & $\begin{array}{c}\text { Responsive- } \\
\text { ness }\end{array}$ & $\begin{array}{c}\text { Criterion } \\
\text { validity }\end{array}$ & $\begin{array}{c}\text { Cross-cultural } \\
\text { validity }\end{array}$ \\
\hline \multicolumn{9}{|l|}{ SOQ } \\
\hline Domino et al., 1982 & & & Poor & Poor & & & & \\
\hline Domino et al., 1988 & Poor & Fair & Poor & & & & & \\
\hline Limbacher \& Domino & & & & Good & & & & \\
\hline \multicolumn{9}{|l|}{$1985-86$} \\
\hline Rogers et al., 1992 & & & & Poor & & & & \\
\hline Rogers et al., 1995 & Poor & Poor & & Poor & & & & \\
\hline Leane \& Shute, 1998 & Poor & & Fair & Poor & Good & & & \\
\hline Domino et al., 2000 & & & & & & & & Poor \\
\hline McAuliffe et al., 2003 & Poor & Fair & & & & & & \\
\hline Beautrais et al., 2004 & & & & Fair & & & & \\
\hline Anderson et al., 2008 & Poor & & & Poor & & & & \\
\hline Vansickle et al., 2016 & & & & Excellent & & & & \\
\hline Lee, 2019 & Poor & Poor & & Poor & & & & Poor \\
\hline \multicolumn{9}{|l|}{ SIQ } \\
\hline Tierney, 1988 & & Poor & & & & Poor & & \\
\hline \multicolumn{9}{|l|}{ SKT } \\
\hline Spirito et al., 1988 & & & & & & Fair & & \\
\hline \multicolumn{9}{|l|}{ SUIATT } \\
\hline Diekstra \& Kerkhof, 1988 & Fair & Fair & Poor & Fair & & & & \\
\hline \multicolumn{9}{|l|}{ KAS } \\
\hline Shaffer et al., 1990 & Poor & Fair & Poor & & & Fair & & \\
\hline \multicolumn{9}{|l|}{ RFOS } \\
\hline Hubbard \& McIntosh, 1992 & & & & & & & Poor & \\
\hline Kolves et al., 2007 & Poor & & & & & & & \\
\hline Voracek et al., 2008 & Fair & & & Fair & & & & \\
\hline \multicolumn{9}{|l|}{ EATSS } \\
\hline Eskin, 1995 & Poor & & Poor & & & & & \\
\hline Eskin, 2004 & & & Poor & Fair & & & & \\
\hline
\end{tabular}




\begin{tabular}{|c|c|c|c|c|c|c|c|c|}
\hline \multirow{2}{*}{\multicolumn{9}{|c|}{$\begin{array}{l}\text { Nader et al., } 2012 \\
\text { PFC-KT }\end{array}$}} \\
\hline & & & & & & & & \\
\hline Mackesy-Amiti et al., 1996 & Fair & Poor & Excellent & Fair & Good & Good & & \\
\hline \multicolumn{9}{|l|}{ ATTS } \\
\hline Renberg \& Jacobsson, 2003 & Good & & Excellent & Good & & & & \\
\hline Mofidi et al., 2008 & Fair & & & Fair & & & & \\
\hline Kodaka et al., 2013 & Fair & & & Fair & & & & \\
\hline Norheim et al., 2013 & Fair & & & Poor & & & & \\
\hline Ji et al., 2016 & Good & Fair & & Good & & & & Fair \\
\hline Siau et al., 2017 & Excellent & & & Excellent & & & & Fair \\
\hline Estrada et al., 2019 & Excellent & & & Excellent & & & & Fair \\
\hline Wallin \& Runeson, 2003 & & & & Good & & & & \\
\hline \multicolumn{9}{|l|}{ SBAQ } \\
\hline Botega et al., 2005 & Good & & Excellent & Good & & & & \\
\hline Botega et al., 2007 & & & & & & Good & & \\
\hline La Guardia et al.,2018 & Poor & & & & Poor & Poor & & \\
\hline Sun et al., 2007 & Poor & & Excellent & & & & & \\
\hline Sun et al., 2011 & Poor & Fair & Poor & & & Fair & & \\
\hline Siau et al;, 2019 & Poor & & Poor & & & & & Fair \\
\hline \multicolumn{9}{|l|}{ CASQ-HK } \\
\hline Lee et al., 2007 & Good & & Excellent & Good & & & & \\
\hline \multicolumn{9}{|l|}{ BIRFFS } \\
\hline Voracek et al., 2007a & Poor & Fair & Poor & Fair & & & Poor & \\
\hline Voracek et al., 2007b & Poor & & Poor & Fair & & & Poor & \\
\hline Voracek et al, 2007c & Poor & Fair & & Fair & & & Poor & \\
\hline Voracek et al, 2008a & Poor & & & Fair & & & Poor & Fair \\
\hline Voracek et al., 2008b & Poor & & & Fair & & & Poor & Fair \\
\hline \multicolumn{9}{|l|}{ ATAS-Q } \\
\hline Ouzouni \& Nakakis, 2009 & Poor & Fair & Fair & Poor & & & & \\
\hline \multicolumn{9}{|l|}{ GK } \\
\hline Bean \& Baber, 2011 & Poor & & Fair & & Fair & Good & & \\
\hline
\end{tabular}


LOSS

Chan et al., 2014

Poor

Rivera-Segarra et al., 2018

Poor

Note. Quality assessment for the measurement error is omitted in this table because none of the scales were concerned. Hyphens stand for unnamed scales

ATAS-Q = Attitude Towards Attempted Suicide-Questionnaire; ATTS = Attitudes toward suicide; BIRFFS =The Beliefs in the Inheritance of Risk Factors for Suicide Scale;

CASQ-HK = Chinese Attitudes Toward Suicide Questionnaire-Hong Kong; EATSS = Eskin's Attitudes Toward Suicide Scale; GK = General knowledge about youth suicide/General knowledge for youth participant; KAS = Knowledge and Attitudes about Suicide; LOSS = Literacy of Suicide Scale; PFC-KT = Preparing for Crisis Knowledge Test; RFOS = Revised Facts on Suicide Quiz; SBAQ = Suicide Behaviour Attitude Questionnaire; SIQ = Suicide Intervention Questionnaire; SKT = Suicide Knowledge Test; SOQ = Suicide Opinion Questionnaire; SUIATT = Suicide Attitude Questionnaire 


\section{Content of the instruments}

Owing to the broad scope of our research algorithm, the selected scales varied in terms of the literacy-related construct they assessed. Some questionnaires merely explored knowledge of suicide, such as the PFC-KT, the LOSS, the RFOS and its variants, the BIRFFS, and the GK. Others referred to the broader notion of attitudes without specification, such as the ATTS, the SUIATT, the Eskin's Attitudes Toward Suicide Scale (EATSS) and the ATAS-Q. Remaining scales combined in varying proportions pure knowledge questions and probes for prejudice or stigmatizing attitudes.

Of the 18 scales we identified, 17 were submitted to content analysis. The SIQ had to be excluded, due to inaccessibility of its detailed content. The different qualitative analytical steps are available in the supplementary Table S1. We extracted 41 unique statements related to knowledge of suicide from scales' items. Merging of kindred notions resulted in 29 core labels, from which we derived 6 thematic homogeneous categories.

The extent to which each scale addressed these labels and themes is summarized in Table 3. The epidemiological theme, which was addressed by 8 scales, encompassed knowledge about the incidence and predictors of suicidal behaviors. The RFOS contained the most complete inquiry of epidemiological knowledge. The second theme dealt with the consequences of media coverage of suicide. It concerned insights that incautious broadcasts of suicide stories may cause imitative behaviors through the Werther effect, or, on the contrary, that responsible articles about individuals who overcame a suicide crisis have been negatively correlated with suicide rates (Papageno effect) (Niederkrotenthaler et al., 2010). Only the ATTS, the CASQ-HK and the PFC-KT inquired knowledge about theses possible consequences, with deeper examination in the PFC-KT. We inferred the 4 remaining themes based on widely-held myths about suicide that we assumed literacy labels would contribute dispelling. The first myth could be formulated as "It is trivial to experience suicidal ideations 
or attempt suicide". It corresponds to knowledge that helps recognizing suicidal behaviors as serious events that warrant attention and help. It was at least partially explored by all scales but the BIRFFS, with greater insights in the ATAS-Q and Sun et al.'s scale. The second myth relates to the presumed unpredictability of suicidal behaviors. It basically encompasses knowledge of warning signs and risks of repetitions. It is the least represented theme among included scales, with only 8 instruments containing related items. Of note, only the SKT deals with the suicide note, and only the SOQ inquiries about the persistent risk after a suicide attempt. The third myth could be stated as follows: "Suicidal behaviors are explainable by a single, readily identifiable cause". This covers knowledge about the mains risk factors of suicidal behaviors (e.g. psychiatric disorder, alcohol dependence, adverse life events), thus promoting multicausal non-deterministic understandings of suicide. In our sample, only the PFC-KT didn't address this myth. By contrast, the Knowledge and Attitudes about Suicide (KAS) (Shaffer et al., 1990) and Sun et al.'s questionnaire were the most complete tools (nonexhaustive, though) in regard to the causal category of knowledge. Finally, the fourth myth corresponded to the common misconception that suicide wouldn't be preventable. Related knowledge concerns the reversibility of the suicidal process, as well as the efficacy and harmlessness of suicide prevention actions. All the scales addressed two or more of the knowledge labels affiliated to this myth, except the BIRFFS, the PFC-KT and the GK that explored none of them. 
Table 3. Content of the scales evaluating knowledge about suicide according to the thematic analysis.

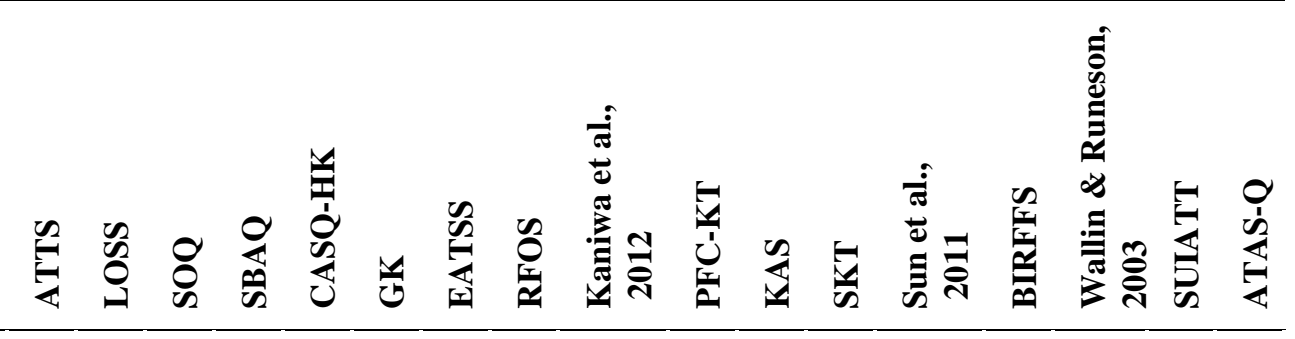

\section{Epidemiology}

Suicide method

Prevalence of suicidal ideation

Epidemiological variations related socio-demographic

characteristics

Incidence of suicide

Suicide/suicide attempt ratio

Epidemiological variations related to socio-economic conditions

Temporal variations of suicide rates

Epidemiological variations related to geographical or natural conditions

\section{Consequences of media coverage of suicide}

It is trivial and harmless to communicate on suicide

Suicide can sometimes be linked to a phenomenon of imitation

Media coverage of suicide can change the risk of suicidal action in vulnerable individuals

\section{Myth 1: "experiencing suicidal thoughts or attempting suicide is}

\section{trivial."}

Having attempted suicide increases the risk of suicide.

Having experienced a trauma or suffering from an incurable condition non-deterministically increases the risk of suicidal ideations
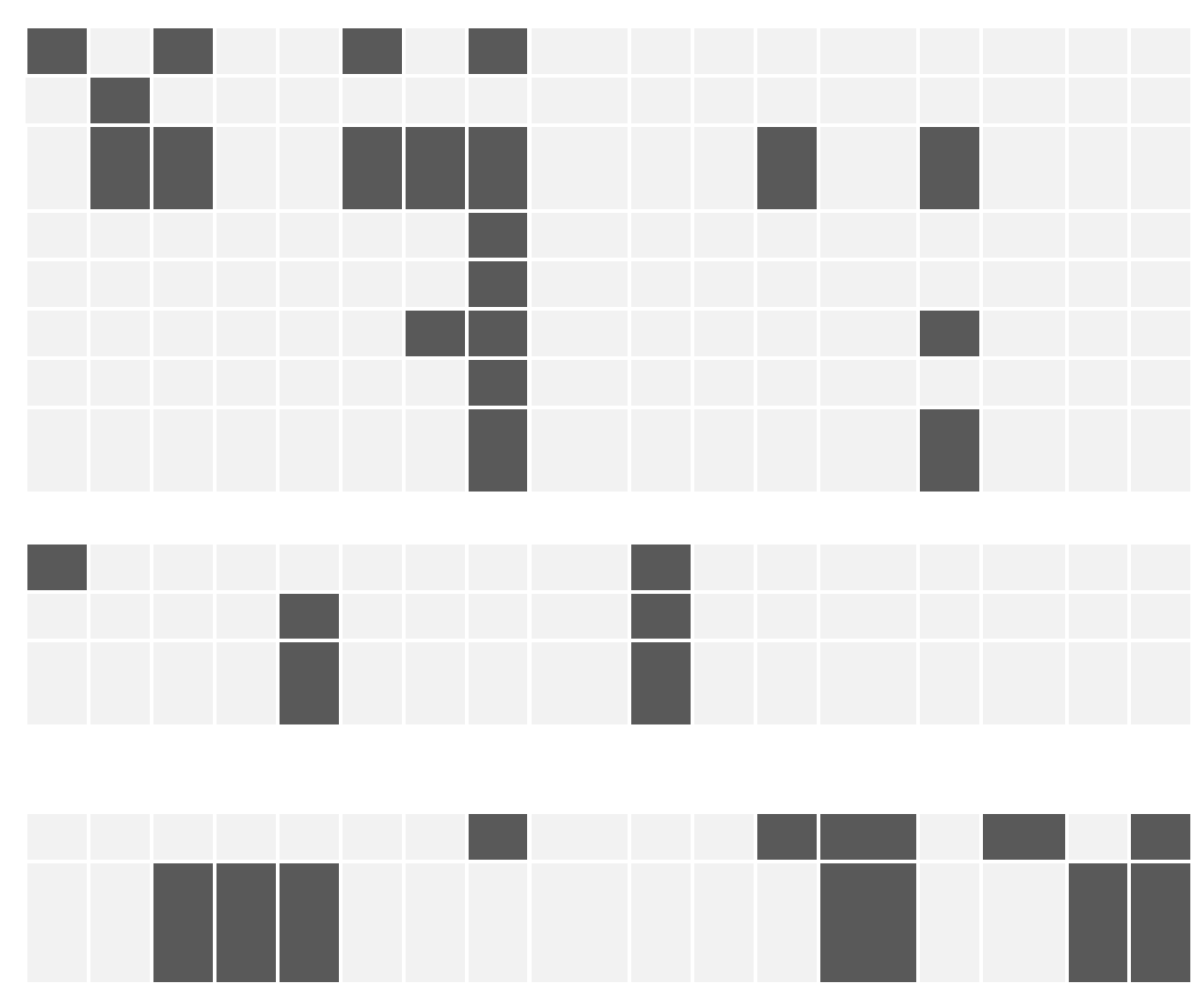
Person who share their suicidal ideations or attempt suicide without dying are at greater risk of suicide

Experiencing suicidal ideation is nontrivial experience

\section{Myth 2. "Suicide or suicide attempts are unpredictable."}

The majority of individuals who attempt suicide share their intention, seek help, change their behavior of leave cues

beforehand

When a person gets better after a suicide attempts, this doesn't necessarily means that there is no more risk

Joyful persons aren't safe from suicide

People who commit suicide may or may not leave a letter

Myth 3. "Suicide can be explained by a single, identifiable cause"

There is a frequent association between alcohol dependence and suicide

There is a hereditary predisposition to suicide

Suicide attempts and suicide result from the repetition of negative life events, especially affecting interpersonal relationships

Suicide also concern religious people

In the vast majority of cases, suicidal ideations, suicide attempts and suicide are associated with a psychiatric disorder

\section{Myth 4. "Suicide isn't preventable"}

Suicide is preventable, and anyone can contribute to prevention efforts

In individuals' subjective experience, suicide is the only option to escape overwhelming problems. It may, but also may not be driven by a conscient wish to die

Talking about suicide may trigger suicidal ideation or suicide attempt

Suicide and suicide attempts are impulsive behaviors, people can quickly change their minds.

A substantial proportion of persons who are suicidal at one point won't have further suicidal ideations along their life.
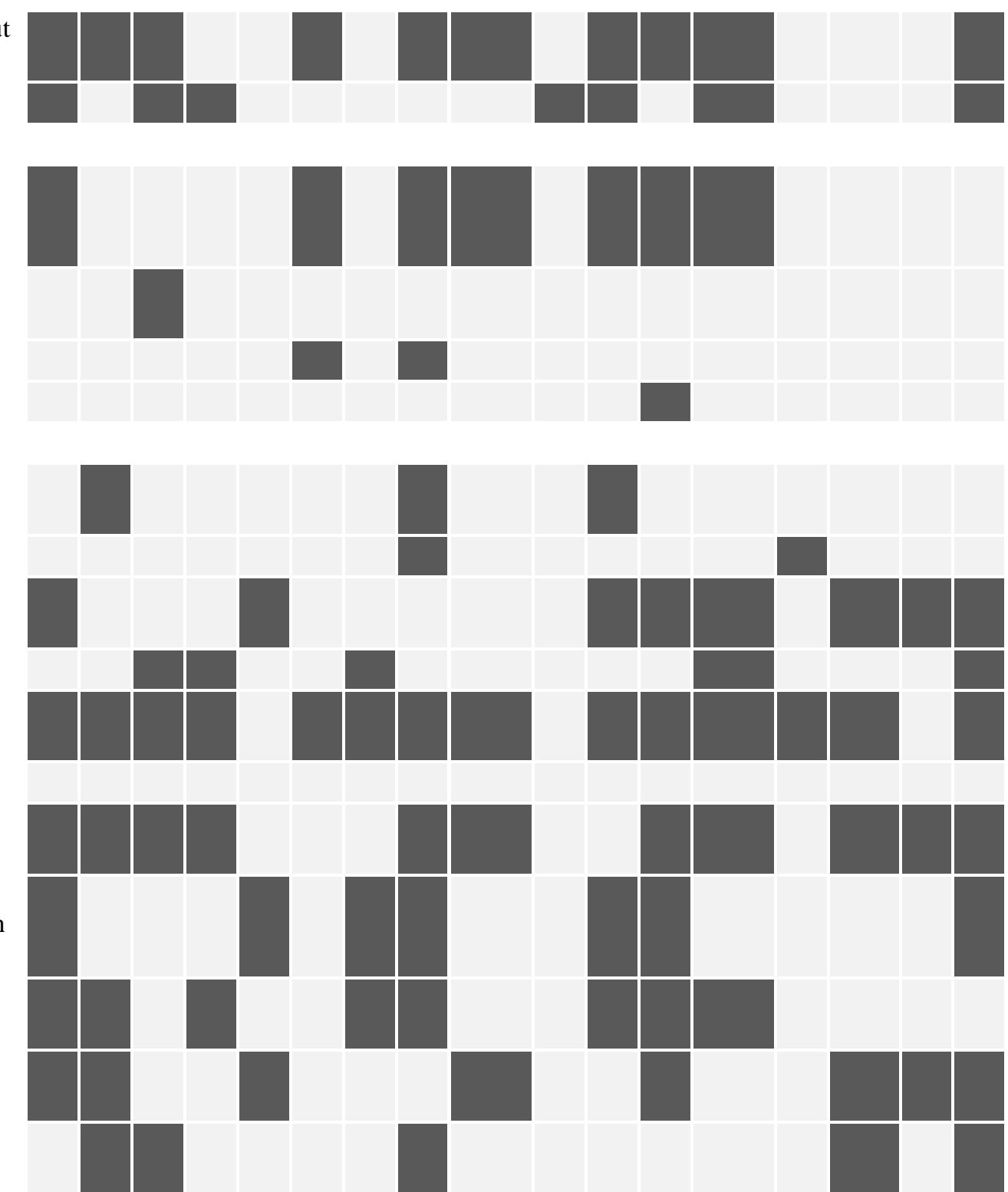
Note. Cells are filled with dark gray when the corresponding thematic item (in line) is covered by the corresponding scale (in column). Unlabelled scales are designated by a reference to the corresponding paper. ATAS-Q. Attitude Towards Attempted Suicide-Questionnaire (Ouzouni \& Nakakis, 2009) ATTS. Attitudes toward suicide (Renberg \& Jacobsson, 2003); BIRFFS. The Beliefs in the Inheritance of Risk Factors for Suicide Scale (Voracek et al., 2007a); CASQ-HK. Chinese Attitudes Toward Suicide QuestionnaireHong Kong (Lee et al., 2007); EATSS. Eskin's Attitudes Toward Suicide Scale (Eskin, 1992); GK. General knowledge about youth suicide/General knowledge for youth participant (Bean \& Baber, 2011); KAS. Knowledge and Attitudes about Suicide (Shaffer et al., 1990); LOSS. Literacy of Suicide Scale (Chan et al., 2014); PFC-KT. Preparing for Crisis - Knowledge Test (Mackesy-Amiti et al., 1996); RFOS. Revised Facts on Suicide Quiz (Hubbard \& McIntosh, 1992); SA. Suicide attempt; SBAQ. Suicide Behaviour Attitude Questionnaire (Botega et al., 2005); SKT. Suicide Knowledge Test (Spirito et al., 1988); SOQ. Suicide Opinion Questionnaire (Domino et al., 1982); SUIATT. Suicide Attitude Questionnaire (Diekstra \& Kerkhof, 1988). 


\section{Discussion}

\section{Summary of the main results}

In the present study, we exhaustively reviewed the scales that, for all or part, were designed to measure knowledge or literacy of suicide. From a metrological point of view, variability of psychometric properties stands out as the most prominent result. The dimensionality of the "knowledge" or "literacy" constructs was found generally high but also labile across scales or validation samples. On average, intra-dimensional internal consistency was modest, reflecting the heterogeneity of the notions under assessment. From an epistemological point of view, the thematic analysis revealed that available scales variably address knowledge about epidemiology, consequences of suicide coverage and 4 common myths about severity, predictability, causality and preventability of suicidal behaviors.

Five scales stand out as acceptable tools to assess literacy of suicide because of their robustness and/or quality of validation: The BIRFFS focuses on a very circumscribed subfield of suicide literacy, namely the knowledge that suicidal behaviors comprise an heritable component; more comprehensive but less specific, the CASQ-HK, the SBAQ and the ATTS tangentially cover knowledge of suicide while focusing on a composite grasp of the concept of attitude; finally the SOQ appraises knowledge of suicide under the notion of stereotypes as a component of the stigma model.

\section{Interpretation of the results}

The notional complexity of knowledge exacerbates a psychometrically-inherent problem, namely how to objectively and rationally delineate abstract clinical concepts. As suggested by our thematic analysis, available scales mostly rely on a data-driven approach, leaving to statistical component analysis the task of finding out coherences in conceptually composite items. However, the intra and inter-scale dimensional lability, as well as the generally poor internal consistencies reveal the lack of theoretical balancing to stabilize and 
optimize the measure of the "knowledge" or "literacy" constructs. Nevertheless, theoretical models have been developed and tested for more than 40 years that could help in differentiating knowledge and literacy from related concepts, but also in specifying the nature and components of the constructs.

Our decision to focus this review on knowledge at the exclusion of prejudice or stigma is based on social cognitive models that articulate these notions as clearly distinct entities. In both Corrigan's stigma models or Link's labeling theory, knowledge is assimilable to stereotypes, which consist in cognitive schemes learnt by most members of a social group to help efficiently categorize and give meaning to socially-relevant information (Corrigan, 2000; Link and Phelan, 2001). When endorsed, incorrect or imprecise core assumptions (that can be related to myths or misconceptions) give raise to hostile emotional responses resulting in prejudice. The last layer of the stigma model consists in discriminatory behaviors that have their roots in both stereotypes and prejudice (Corrigan et al., 2005). In psycho-social and cognitive conceptualizations, the term "attitude" appears less-well defined, indifferently used as an equivalent to prejudice ("negative attitude") or stigmatizing behavior ("stigmatizing attitudes"). In other acceptations, "attitude" more broadly encompasses the different layers of the stigma model. This conceptual blurry is an obstacle for valid measure, which is reflected by the modest constancy of scales appealing to the notion.

Even when distinguished from neighboring notions, knowledge of suicide doesn't appear as a homogeneous construct. From an epistemological point of view, it is noteworthy that the knowledge categories about suicide we identified from the thematic analysis are not all of the same nature. On the one hand, knowledge about epidemiology and suicide contagion are easily confirmable or falsified. Empirical data exist that can provide a firm and definitive standard to inform the truthfulness of related statements. Such knowledge is purely objective and follows a binary logic : a proposition can only be absolutely true of false. For instance, 
the claim that broadcasts of suicide stories doesn't influence suicide rates can be definitely refuted, based on robust empirical evidence (Niederkrotenthaler et al., 2020). On the other hand, so-called "myths" about suicide appeal to more complex and composite knowledge. Suicide is not only a scientific or a social objectbut also relates to clinical matters. As such, it should be appraised under the evidence-based principlewhich consider scientific evidence and individual clinical experience to be inseparable and both as legitimate. Although necessary, the literature might not be sufficient to endorse the truthfulness of some common statements about suicide. For instance, the items we identified about the seriousness, causality, preventability and predictability of suicidal behaviors relate to complex and integrated knowledge. They come with an irreducible subjective component that makes their veracity at least partially relative. This doesn't mean that misconceptions about suicide cannot be refuted, though. It only suggests that contrary to purely objective knowledge, the reliability of some conceptions of suicide may rather be informed by clinical expert consensus prevailing at a given period. Such consensual standard acknowledges that endorsement of clinical statements related to suicide can be dimensional (for instance, one can "totally disagree" or "tend to disagree" with the idea that suicide is the expression of an ultimate freedom). Still, it allows for asserting that some statements should be considered true and some others false.

Finally, the notion of literacy doesn't totally overlap with that of knowledge. Stemming from the general public health culture, literacy has been defined as "the ability to access, understand, and communicate information to engage with the demands of different health contexts to promote good health across life course". In line with the conceptualization proposed by the World Health Organization (WHO, 2014), Wei al. suggests that mental literacy programs address 3 related issues: knowledge, attitude and help-seeking efficacy (Wei et al., 2013). Translated into the suicide prevention realm, literacy encompasses a wide range of phenomena, including knowledge, clinical skills, help-seeking capacities, 
interpersonal abilities and self-efficacy. As such, literacy poses the fully-fledged evaluation challenge of differentiating pure "knowing" from "knowing how to do" and "knowing how to be". As they are of different nature, these components may require separate assessment methods with specific objectives. In our review, we attempted to isolate the cognitive or representational component of knowledge exclusive of suicide prevention skills, with the idea that the evaluation of knowledge and skills can complement each other only if taken as distinct entities.

\section{Confrontation with the literature}

To our knowledge, only three reviews have previously sought to inventory the most relevant instruments to measure knowledge of suicide, either explicitly identified as such of under the label "attitudes".

Adopting a broader clinical scope than ours, Range and Knott included scales about attitudes towards suicide, but also suicidal risk assessment tools and inquiries of reasons to die by suicide (Range and Knott, 1997). Among the relevant attitudinal measures, Range and Knott mention the SOQ which is consistent with our findings, but also the Suicide Intervention Response Inventory (SIRI). This latter instrument was developed to assess the ability of counsellors to respond appropriately to suicidal patients. Rather than theoretical knowledge, the SIRI uses situational vignettes to test the respondents' prevention skills.

Closer to our objective, the review from Kodaka and colleagues reaches the same conclusion about the interest of SOQ and ATTS (Kodaka et al., 2011). It is however important to note that the authors included all the possible kinds of tools that evaluate attitudes, including vignettes, scenarios, videos, etc. This adds a level of docimological complexity that we preferred to neutralize by focusing on self-administered scales. Although the question about how to test knowledge has its own interest, we considered that a clarification about what is knowledge was a necessary preliminary step to achieve. 
Finally, Ghasemi et al. reviewed 14 published validated scales about attitudes toward suicide (Ghasemi et al., 2015). Seven of them were congruent with what we found, as clearly probing cognitive schemes and representations about suicide. Interestingly, the authors refer to myths, beliefs and stereotypes along the notion of knowledge, and highlight the importance of taking in account the societal and cultural anchoring. However, 7 scales identified by Ghasemi et al. didn't match our inclusion criteria. This could be explained by our more restrictive strategy when balancing the sake of exhaustivity with the objective of notional specification.

\section{Limitations}

Some limitations should be taken into account before considering the relevance of our results. The most important is inherent to the very purpose of the study. Trying to delineate a construct from the scales that already have been proposed to measure it poses a chicken-and-egg problem. Indeed, a preconception of knowledge of suicide was necessary to set the inclusion and exclusion criteria, which might appear contradictory with our effort in precising how knowledge of suicide is understood by scholars. Relatedly, our decision to exclude from the analysis the measures of stigma, prejudice or skills about suicide can raise questions. However, the reader should bear in mind that our goal was not to uncover the absolute definition of knowledge about suicide, but rather to help stabilize the concept by combining a synthesis of data-driven evidence and theoretical contributions. Our non-naïve approach, based on a basic pre-review of the literature, allowed to put a priori constraints on the research. This facilitated the epistemological convergence of our results and enabled a gain in conceptual precision. 


\section{Conclusions}

Future work on how to measure knowledge of suicide should clearly specify which kind of construct is under study. Such preliminary theoretical work appears necessary in order to optimize both the comprehensibility and interpretability of any assessment. In addition, measurement and rating methods should be adapted to each type of knowledge construct. In particular, a special effort should be made to evaluate the truthfulness of knowledge statements. Such specification is not only necessary to increase psychometric quality and validity, it also to improves the targeting of prevention strategies related to suicide. 


\section{Acknowledgment}

We thank Lucie Sharkey for having kindly accepted to perform the English editing of the manuscript.

\section{Funding}

This research did not receive any specific grant from funding agencies in the public, commercial, or not-for-profit sectors.

\section{Conflicts of interest}

All other authors declare that they have no conflicts of interest. 


\section{References}

Anderson, A.L., Lester, D., and Rogers, J.R. (2008). A Psychometric Investigation of the Suicide Opinion Questionnaire. Death Stud. 32, 924-936.

Bean, G., and Baber, K.M. (2011). Connect: An Effective Community-Based Youth Suicide Prevention Program. Suicide Life. Threat. Behav. 41, 87-97.

Beautrais, A.L., John Horwood, L., and Fergusson, D.M. (2004). Knowledge and attitudes about suicide in 25-year-olds. Aust. N. Z. J. Psychiatry 38, 260-265.

Botega, N.J., Reginato, D.G., Silva, S.V. da, Cais, C.F. da S., Rapeli, C.B., Mauro, M.L.F., Cecconi, J.P., and Stefanello, S. (2005). Nursing personnel attitudes towards suicide: the development of a measure scale. Rev. Bras. Psiquiatr. 27, 315-318.

Botega, N.J., Silva, S.V., Reginato, D.G., Rapeli, C.B., Cais, C.F.S., Mauro, M.L.F., Stefanello, S., and Cecconi, J.P. (2007). Maintained Attitudinal Changes in Nursing Personnel After a Brief Training on Suicide Prevention. Suicide Life. Threat. Behav. 37, 145-153.

Chan, W.I., Batterham, P., Christensen, H., and Galletly, C. (2014). Suicide literacy, suicide stigma and help-seeking intentions in Australian medical students. Australas. Psychiatry 22, 132-139.

Cimini, M.D., Rivero, E.M., Bernier, J.E., Stanley, J.A., Murray, A.D., Anderson, D.A., Wright, H.R., and Bapat, M. (2014). Implementing an audience-specific small-group gatekeeper training program to respond to suicide risk among college students: a case study. J. Am. Coll. Health J ACH 62, 92-100.

Corrigan, P.W. (2000). Mental Health Stigma as Social Attribution: Implications for Research Methods and Attitude Change. Clin. Psychol. Sci. Pract. 7, 48-67.

Corrigan, P.W., Kerr, A., and Knudsen, L. (2005). The stigma of mental illness: Explanatory models and methods for change. Appl. Prev. Psychol. 11, 179-190.

Cross, W.F., Seaburn, D., Gibbs, D., Schmeelk-Cone, K., White, A.M., and Caine, E.D. (2011). Does Practice Make Perfect? A Randomized Control Trial of Behavioral Rehearsal on Suicide Prevention Gatekeeper Skills. J. Prim. Prev. 32, 195.

Diekstra, R.F.W., and Kerkhof, A.J.F.M. (1988). Attitudes Toward Suicide: Development of a Suicide Attitude Questionnaire (SUIATT). In Current Issues of Suicidology, (Springer, Berlin, Heidelberg), pp. 462-476.

Domino, G., Moore, D., Westlake, L., and Gibson, L. (1982). Attitudes toward suicide: A factor analytic approach. J. Clin. Psychol. 38, 257-262.

Domino, G., Macgregor, J.C., and Hannah, M.T. (1988). Collegiate Attitudes toward Suicide: New Zealand and United States. OMEGA - J. Death Dying 19, 351-364.

Domino, G., Su, S., and Shen, D. (2000). Cross-Cultural Investigation of a New Set of Scales for the Suicide Opinion Questionnaire. OMEGA - J. Death Dying 41, 307-321.

Eskin, M. (1995). Adolescents' attitudes toward suicide, and a suicidal peer: A comparison between Swedish and Turkish high school students. Scand. J. Psychol. 36, 201-207.

Estrada, C.A.M., Nonaka, D., Gregorio, E.R., Leynes, C.R., del Castillo, R.T., Hernandez, P.M.R., Hayakawa, T., and Kobayashi, J. (2019). Suicidal ideation, suicidal behaviors, and attitudes towards suicide of adolescents enrolled in the Alternative Learning System in Manila, Philippines-a mixed methods study. Trop. Med. Health 47, 22.

Ghasemi, P., Shaghaghi, A., and Allahverdipour, H. (2015). Measurement Scales of Suicidal Ideation and Attitudes: A Systematic Review Article. Health Promot. Perspect. 5, 156-168. 
Harrod, C.S., Goss, C.W., Stallones, L., and DiGuiseppi, C. (2014). Interventions for primary prevention of suicide in university and other post-secondary educational settings. Cochrane Database Syst. Rev. CD009439.

Hubbard, R.W., and McIntosh, J.L. (1992). Integrating Suicidology into Abnormal Psychology Classes: The Revised Facts on Suicide Quiz. Teach. Psychol. 19, 163-166.

Ji, N.-J., Hong, Y.-P., and Lee, W.-Y. (2016). Comprehensive psychometric examination of the attitudes towards suicide (ATTS) in South Korea. Int. J. Ment. Health Syst. 10, 2.

Jollant, F., Bellivier, F., Leboyer, M., Astruc, B., Torres, S., Verdier, R., Castelnau, D., Malafosse, A., and Courtet, P. (2005). Impaired Decision Making in Suicide Attempters. Am. J. Psychiatry 162, 304310.

Jollant, F., Lawrence, N.L., Olié, E., Guillaume, S., and Courtet, P. (2011). The suicidal mind and brain: A review of neuropsychological and neuroimaging studies. World J. Biol. Psychiatry 12, 319-339.

Jorm, A.F. (2000). Mental health literacy. Public knowledge and beliefs about mental disorders. Br. J. Psychiatry J. Ment. Sci. 177, 396-401.

Kaniwa, I., Kawanishi, C., Suda, A., and Hirayasu, Y. (2012). Effects of Educating Local Government Officers and Healthcare and Welfare Professionals in Suicide Prevention. Int. J. Environ. Res. Public. Health 9, 712-721.

Kato, T.A., Suzuki, Y., Sato, R., Fujisawa, D., Uehara, K., Hashimoto, N., Sawayama, Y., Hayashi, J., Kanba, S., and Otsuka, K. (2010). Development of 2-hour suicide intervention program among medical residents: First pilot trial. Psychiatry Clin. Neurosci. 64, 531-540.

Kodaka, M., Postuvan, V., Inagaki, M., and Yamada, M. (2011). A systematic review of scales that measure attitudes toward suicide. Int. J. Soc. Psychiatry 57, 338-361.

Kodaka, M., Inagaki, M., Poštuvan, V., and Yamada, M. (2013). Exploration of factors associated with social worker attitudes toward suicide. Int. J. Soc. Psychiatry 59, 452-459.

Kõlves, K., Tran, U.S., and Voracek, M. (2007). Knowledge about Suicide and Local Suicide Prevalence: Comparison of Estonia and Austria. Percept. Mot. Skills 105, 3-7.

La Guardia, A.C., Cramer, R.J., Brubaker, M., and Long, M.M. (2019). Community Mental Health Provider Responses to a Competency-Based Training in Suicide Risk Assessment and Prevention. Community Ment. Health J. 55, 257-266.

Leane, W., and Shute, R. (1998). Youth Suicide: The Knowledge and Attitudes of Australian Teachers and Clergy. Suicide Life. Threat. Behav. 28, 165-173.

Lee, W.-Y. (2019). Comparisons in the attitudes toward suicide among urban residents, lawmakers and community mental health workers in South Korea. Psychiatry Res. 276, 278-282.

Lee, S., Tsang, A., Li, X., Phillips, M.R., and Kleinman, A. (2007). Attitudes Toward Suicide Among Chinese People in Hong Kong. Suicide Life. Threat. Behav. 37, 565-575.

Limbacher, M., and Domino, G. (1986). Attitudes toward Suicide among Attempters, Contemplators, and Nonattempters. OMEGA - J. Death Dying 16, 325-334.

Link, B.G., and Phelan, J.C. (2001). Conceptualizing Stigma. Annu. Rev. Sociol. 27, 363-385.

Lytle, M.C., Silenzio, V.M.B., and Caine, E.D. (2016). Are There Still Too Few Suicides to Generate Public Outrage? JAMA Psychiatry 73, 1003-1004.

Mackesy-Amiti, M.E., Fendrich, M., Libby, S., Goldenberg, D., and Grossman, J. (1996). Assessment of Knowledge Gains in Proactive Training for Postvention. Suicide Life. Threat. Behav. 26, 161-174. 
McAuliffe, C., Corcoran, P., Keeley, H.S., and Perry, I.J. (2003). Risk of Suicide Ideation Associated with Problem-Solving Ability and Attitudes Toward Suicidal Behavior in University Students. Crisis J. Crisis Interv. Suicide Prev. 24, 160-167.

Mofidi, N., Ghazinour, M., Salander-Renberg, E., and Richter, J. (2008). Attitudes towards suicide among Kurdish people in Iran. Soc. Psychiatry Psychiatr. Epidemiol. 43, 291-298.

Moher, D., Liberati, A., Tetzlaff, J., Altman, D.G., Group, P., and others (2009). Preferred reporting items for systematic reviews and meta-analyses: the PRISMA statement. PLoS Med 6, e1000097.

Mokkink, L.B., Terwee, C.B., Patrick, D.L., Alonso, J., Stratford, P.W., Knol, D.L., Bouter, L.M., and de Vet, H.C.W. (2010). The COSMIN checklist for assessing the methodological quality of studies on measurement properties of health status measurement instruments: an international Delphi study. Qual. Life Res. 19, 539-549.

Niederkrotenthaler, T., Voracek, M., Herberth, A., Till, B., Strauss, M., Etzersdorfer, E., Eisenwort, B., and Sonneck, G. (2010). Role of media reports in completed and prevented suicide: Werther v. Papageno effects. Br. J. Psychiatry 197, 234-243.

Niederkrotenthaler, T., Braun, M., Pirkis, J., Till, B., Stack, S., Sinyor, M., Tran, U.S., Voracek, M., Cheng, Q., Arendt, F., et al. (2020). Association between suicide reporting in the media and suicide: systematic review and meta-analysis. BMJ m575.

Norheim, A.B., Grimholt, T.K., and Ekeberg, Ø. (2013). Attitudes towards suicidal behaviour in outpatient clinics among mental health professionals in Oslo. BMC Psychiatry 13, 90.

O'Carroll, P.W., and Potter, L.B. (1994). Suicide contagion and the reporting of suicide: recommendations from a national workshop. United States Department of Health and Human Services. MMWR Recomm. Rep. Morb. Mortal. Wkly. Rep. Recomm. Rep. Cent. Dis. Control 43, 9-17.

Ortiz, P., and Khin Khin, E. (2018). Traditional and new media's influence on suicidal behavior and contagion. Behav. Sci. Law 36, 245-256.

Ouzouni, C., and Nakakis, K. (2009). Attitudes towards attempted suicide: The development of a measurement tool. Health Sci. J. 3(4), 222-231.

Range, L.M., and Knott, E.C. (1997). Twenty Suicide Assessment Instruments: Evaluation and Recommendations. Death Stud. 21, 25-58.

Renberg, E.S., and Jacobsson, L. (2003). Development of a Questionnaire on Attitudes Towards Suicide (ATTS) and Its Application in a Swedish Population. Suicide Life. Threat. Behav. 33, 52-64.

Robinson, J., Cox, G., Bailey, E., Hetrick, S., Rodrigues, M., Fisher, S., and Herrman, H. (2015). Social media and suicide prevention: a systematic review. Early Interv. Psychiatry n/a-n/a.

Rogers, J.R., and DeShon, R.P. (1992). A Reliability Investigation of the Eight Clinical Scales of the Suicide Opinion Questionnaire. Suicide Life. Threat. Behav. 22, 428-441.

Rogers, J.R., and DeShon, R.P. (1995). Cross-Validation of the Five-Factor Interpretive Model of the Suicide Opinion Questionnaire. Suicide Life. Threat. Behav. 25, 305-309.

Ryan, K., Tindall, C., and Strudwick, G. (2017). Enhancing Key Competencies of Health Professionals in the Assessment and Care of Adults at Risk of Suicide Through Education and Technology. Clin. Nurse Spec. CNS 31, 268-275.

SAVE (2019). Suicide Prevention, Information, and Awareness.

Shaffer, D., Vieland, V., Garland, A., Rojas, M., Underwood, M., and Busner, C. (1990). Adolescent Suicide Attempters: Response to Suicide-Prevention Programs. JAMA 264, 3151-3155. 
Shepard, D.S., Gurewich, D., Lwin, A.K., Reed, G.A., and Silverman, M.M. (2016). Suicide and Suicidal Attempts in the United States: Costs and Policy Implications. Suicide Life. Threat. Behav. 46, 352-362.

Siau, C.S., Wee, L.-H., Ibrahim, N., Visvalingam, U., and Wahab, S. (2017). Cross-Cultural Adaptation and Validation of the Attitudes Toward Suicide Questionnaire Among Healthcare personnel in Malaysia. Inq. J. Health Care Organ. Provis. Financ. 54, 0046958017707295.

Spirito, A., Overholser, J., Ashworth, S., Morgan, J., and Benedict-drew, C. (1988). Evaluation of a Suicide Awareness Curriculum for High School Students. J. Am. Acad. Child Adolesc. Psychiatry 27, 705-711.

Strauss, A., and Corbin, J. (2006). Grounded Theory Methodology. An Overview. In The Blackwell Encyclopedia of Sociology., (Oxford: Blackwell Publishing), pp. 273-295.

Sun, F.-K., Long, A., and Boore, J. (2007). The attitudes of casualty nurses in Taiwan to patients who have attempted suicide. J. Clin. Nurs. 16, 255-263.

Tierney, R.J. (1994). Suicide intervention training evaluation: a preliminary report. Crisis 15, 69-76.

VanSickle, M., Werbel, A., Perera, K., Pak, K., DeYoung, K., and Ghahramanlou-Holloway, M. (2016). Principal Component Analysis of the Suicide Opinion Questionnaire in a U.S. Military Sample of Marine Corps Non-Commissioned Officers. Mil. Med. 181, 672-679.

Voracek, M., Loibl, L.M., and Sonneck, G. (2007a). Beliefs in the Inheritance of Risk Factors for Suicide Scale: development, reliability, stability, and convergent and discriminant validity. Psychol. Rep. 101, 107-116.

Voracek, M., Loibl, L.M., Egle, J., Schleicher, S., and Sonneck, G. (2007b). Correlates, Item-Sequence Invariance, and Test-Retest Reliability of the Beliefs in the Inheritance of Risk Factors for Suicide Scale (BIRFSS). Psychol. Rep. 101, 1107-1117.

Voracek, M., Egle, J., Schleicher, S., Loibl, L.M., and Sonneck, G. (2007c). The Beliefs in the Inheritance of Risk Factors for Suicide Scale (Birfss): Further Results on Demographic Correlates, Dimensionality, Reliability, and Validity. OMEGA - J. Death Dying 55, 279-296.

Voracek, M., Tran, U.S., and Sonneck, G. (2008a). Psychometric Properties of the Revised Facts on Suicide Quiz in Austrian Medical and Psychology Undergraduates. Death Stud. 32, 937-950.

Voracek, M., Loibl, L.M., Swami, V., Vintilă, M., Kõtlves, K., Sinniah, D., Pillai, S.K., Ponnusamy, S., Sonneck, G., Furnham, A., et al. (2008b). The Beliefs in the Inheritance of Risk Factors for Suicide Scale (BIRFSS): Cross-Cultural Validation in Estonia, Malaysia, Romania, the United Kingdom, and the United States. Suicide Life. Threat. Behav. 38, 688-698.

Voracek, M., Fisher, M.L., Loibl, L.M., Tan, H., and Sonneck, G. (2008c). Beliefs about the genetics of suicide in Canadian students: Cross-language validation of the Beliefs in the Inheritance of Risk Factors for Suicide Scale (BIRFSS). Psychiatry Clin. Neurosci. 62, 271-278.

Wallin, U., and Runeson, B. (2003). Attitudes towards suicide and suicidal patients among medical students. Eur. Psychiatry 18, 329-333.

Wei, Y., Hayden, J.A., Kutcher, S., Zygmunt, A., and McGrath, P. (2013). The effectiveness of school mental health literacy programs to address knowledge, attitudes and help seeking among youth. Early Interv. Psychiatry 7, 109-121.

WHO (2014). Preventing suicide: a global imperative (World Health Organization).

WHO (2015). World Health Statistics (World Health Organization).

WHO (2016). The top 10 causes of death (World Health Organization). 
